\title{
Investigation of droplet dynamics in a convective cloud using a Lagrangian cloud model
}

\author{
Junghwa Lee $\cdot$ Yign Noh $\cdot$ Siegfried Raasch $\cdot$ \\ Theres Riechelmann $\cdot$ Lian-Ping Wang
}

Received: 9 August 2013/Accepted: 24 January 2014/Published online: 16 February 2014

(C) The Author(s) 2014. This article is published with open access at Springerlink.com

\begin{abstract}
A precipitating convective cloud is simulated successfully using the Lagrangian cloud model, in which the flow field is simulated by large eddy simulation and the droplets are treated as Lagrangian particles, and the results are analyzed to investigate precipitation initiation and to examine the parameterization of cloud microphysics. It is found that raindrops appear initially near the cloud top, in which strong turbulence and broadened droplet spectrum are induced by the entrainment of dry air, but high liquidwater mixing ratio is maintained within cloud parts because of insufficient mixing. Statistical analysis of the downward vertical velocity of a droplet $W$ reveals that the transition from cloud droplets to raindrops occurs in the range $20 \mu \mathrm{m}<r<100 \mu \mathrm{m}$, while the variation of $W$ depends on turbulence as well as the droplet radius $r$. The general pattern of the raindrop size distribution is found to be consistent with the Marshall-Palmer distribution. The precipitation flux can be underestimated substantially, if
\end{abstract}

Responsible editor: R. Roebeling.

J. Lee $\cdot$ Y. Noh $(\bowtie)$

Department of Atmospheric Sciences, Yonsei University,

50 Yonsei-ro, Seodaemun-gu, Seoul 120-749, Korea

e-mail: noh@yonsei.ac.kr

S. Raasch - T. Riechelmann

Institute of Meteorology and Climatology, Leibniz Universität

Hannover, Herrenhaeuser Str. 2, 30419 Hannover, Germany

L.-P. Wang

Department of Mechanical Engineering, University of Delaware,

Newark, DE 19716-3140, USA

L.-P. Wang

Key Laboratory of Coal Combustion, Huazhong University

of Science and Technology, 1037 Luoyu Road,

430074 Wuhan, China the terminal velocity $w_{\mathrm{s}}$ is used instead of $W$, but it is not sensitive to the choice of the critical droplet radius dividing cloud drops and raindrops. It is also found that precipitation starts earlier and becomes stronger if the effect of turbulence is included in the collection kernel.

\section{Introduction}

Simulations of clouds have been carried out traditionally by Eulerian models; either bulk models, which calculate a few numbers of bulk variables, or spectral bin models, which calculate the evolution of droplet spectra (see, e.g., Houze 1993). Although these models have been successful to simulate the cloud motion, there are many cloud processes that can be better represented by the Lagrangian cloud model (LCM), in which the flow field is simulated by large eddy simulation (LES), and the droplets are treated as Lagrangian particles; for example, the initiation of precipitation and its downward flux, the time history of individual droplets, the condensational growth of a droplet through inhomogeneous environment, etc.

The Langrangian approach has often been taken to investigate the condensational growth of a droplet (Vaillancourt et al. 2002; Lasher-Trapp et al. 2005; Lanotte et al. 2009; Sidin et al. 2009; Cooper et al. 2013) or to simulate ice particles (Sölch and Kärcher 2010). The development of LCM has not been achieved until recently, however, because of the difficulties of handling an extremely large number of droplets and the collision process, and only a few attempts have been reported so far (Andrejczuk et al. 2008, 2010; Shima et al. 2009; Riechelmann et al. 2012; Arabas and Shima 2013). The concept of an ensemble of 'super-droplets' is used in all LCM, where a super-droplet, the terminology 
coined by Shima et al. (2009), represents a large number of real droplets of a given size.

Collision schemes are developed with an approach either to represent individual collision events (Shima et al. 2009; Arabas and Shima 2013) or to create the collision process equivalent to that in the spectral bin model (Andrejczuk et al. 2010; Riechelmann et al. 2012). Shima et al. (2009) and Arabas and Shima (2013) used a Monte Carlo scheme for the collision of simulated droplets, in which collisions between randomly sampled pairs of droplets are calculated, and Andrejczuk et al. (2010) used a scheme in which collisions are assumed to occur between superdroplets within grid boxes subdivided by the droplet size, and new super-droplets are created as the outcome of collisions. On the other hand, Riechelmann et al. (2012) introduced a statistical approach, in which the growth of a super-droplet by collision/coalescence is calculated in terms of the background droplet spectrum and turbulence. The outcome is then described in terms of the modification of both the radius and the weighting factor of the superdroplet. Here the weighting factor, which represents the number of contributing real droplets to a super-droplet, differs for each super-droplet, and changes with time.

Riechelmann et al. (2012) applied the LCM to the simulations of an idealized single cloud and shallow cumulus convection, under the condition corresponding to the Barbados Oceanographic and Meteorological Experiment (BOMEX) (Holland and Rasmusson 1973). They showed that the general features of shallow cumulus clouds could be reproduced successfully in agreement with traditional bulk model results. The droplet spectra simulated by the LCM, using the collection kernels with and without the effects of turbulence (Hall 1980; Ayala et al. 2008a, b), resemble the results of a spectral bin model (Xue et al. 2008). Furthermore, the LCM results of shallow cumulus convection are in good agreement with those from the LES intercomparison under the same condition (Siebesma et al. 2003). The sensitivity tests of LCM to two important parameters of the model, the time step and the number of super-droplets, have also been carried out to investigate the convergence of the LCM solution.

In the present work, using the LCM developed by Riechelmann et al. (2012), we show that LCM can reproduce realistically the evolution of a precipitating convective cloud, including the distributions of vertical velocity, dissipation rate, liquid water mixing ratio, and droplet spectrum within a cloud at various stages of cloud development and the formation of raindrops. The results from the LCM are used to clarify precipitation initiation and to examine the parameterization of cloud microphysics such as the vertical velocity of droplets, the raindrop size distribution, and the precipitation flux. With this work we hope to show that LCM has a potential to become a powerful tool for understanding the cloud process and for developing its parameterization.

\section{Model description}

We used the LCM developed by Riechelmann et al. (2012), to which one can refer for a complete description of the model and the numerical method. Here only the basic framework of the model is discussed.

In order to handle an extremely large number of droplets in a cloud, the concept of a super-droplet is introduced. Each super-droplet represents a large number of real droplets of a given size. The number of real droplets belonging to a super-droplet of radius $r_{n}$ is called the 'weighting factor' $A_{n}$. In the present model $A_{n}$ differs for each super-droplet and changes with time. For a given grid box of volume $\Delta V$, the mixing ratio $q_{l}$ is calculated by

$q_{l}=\frac{1}{\rho_{0}} \frac{\rho_{1}}{\Delta V} \sum_{n=1}^{N} A_{n} \frac{4}{3} \pi r_{n}^{3}$,

where $\rho_{0}$ and $\rho_{1}$ are the density of air and liquid water and $N$ is the number of super-droplets in the grid box.

Since $\rho_{0} / \rho_{1}<<1$, the motion of each super-droplet is simulated by

$\frac{\mathrm{d} V_{i}}{\mathrm{~d} t}=\frac{1}{\tau_{p}}\left(u_{i}-V_{i}\right)-\delta_{i 3}\left(1-\frac{\rho_{0}}{\rho_{1}}\right) g$,

where $V_{i}\left(=\mathrm{d} X_{i} / \mathrm{d} t\right)$ is the velocity of the super-droplet, $X_{i}$ is its position, $\tau_{p}$ is the droplet relaxation time, and $u_{i}$ is the fluid velocity at the droplet position. Note that, contrary to the Eulerian model, each droplet moves differently from each other and from the surrounding fluid.

The inertial response time $\tau_{p}$ is calculated using the nonlinear drag law (Clift et al. 1978):

$\tau_{p}^{-1}=\frac{9 v \rho_{0}}{2 r^{2} \rho_{1}}\left(1+0.15 R e_{p}^{0.687}\right)$ with

$\operatorname{Re}_{p}=r \frac{2 r \cdot|\vec{u}(\vec{X})-\vec{V}|}{v}$.

In the absence of the fluid velocity $\left(u_{i}=0\right)$ in $(2)$, the downward velocity of a droplet $W\left(=-V_{3}\right)$ approaches the terminal velocity $w_{s}$.

The flow is simulated by an LES model based on the nonhydrostatic incompressible Boussinesq equation, and the equations of the fluid phase, i.e., the conservation of momentum, mass, energy, and moisture, are essentially the same as other Eulerian LES models of a cloud. The sink/ source terms through condensation/evaporation in the equations of specific humidity $q$ and potential temperature $\theta$ are directly determined by the change of $q_{l}$ in (1), however. The droplet growth due to the condensation/evaporation of each super-droplet is calculated using the equation suggested by Mason (1971), in which the solution and curvature effects on the droplet's equilibrium vapor pressure are neglected. The radius of all super-droplets is initially given by 
$r=1 \mu \mathrm{m}$, which corresponds to the typical size of the smallest droplet/activated cloud condensation nuclei $(\mathrm{CCN})$. It also corresponds to the typical size of the smallest bin in spectral bin models (Ziegler 1985; Khairoutdinov and Kogan 2000). Droplets outside of a cloud experience evaporation, but their minimum radii are maintained at $r=0.1 \mu \mathrm{m}$.

In order to calculate the droplet growth by collision/ coalescence, a statistical approach is taken in which the growth of a super-droplet, arising from a large number of collision events of droplets belonging to the super-droplet, is calculated based on the background droplet spectrum and turbulence, using the collection kernel. Here the background droplet spectrum is evaluated by counting all superdroplets existing in the grid box to which the corresponding super-droplet belongs. The outcome is then described in terms of the modification of the radius $\left(r_{n}\right)$ and the weighting factor $\left(A_{n}\right)$ of each droplet. For this purpose, the growth of $r_{n}$ is calculated by applying the collection kernel using the stochastic growth equation (e.g., Rogers and Yau 1989), and the decrease of $A_{n}$, representing the loss of real droplets within a super-droplet resulting from the coalescence to other bigger super-droplets, is calculated in a similar way to a spectral bin model.

The collection kernel used in the present model is equivalent to that used for a spectral bin model. The collection kernel developed by Ayala et al. (2008b) and Wang and Grabowski (2009) is used (hereafter the AW kernel), in which the effects of turbulence from relative velocity, preferential concentration, and the enhanced collision efficiency are parameterized as a function of the dissipation rate $\varepsilon$ and the Taylor-microscale Reynolds number. The Hall kernel (Hall 1980), which considers only gravitational collision, is also used for comparison. In this paper the results are from the LCM with the AW kernel, unless it is stated otherwise explicitly. Only the droplet size distribution within a grid box, not the exact locations of droplets within a grid, is relevant to the droplet growth from collision in the present model, as in a spectral model. Momentum exchange between droplets and fluid is not considered.

Results from LES and direct numerical simulation (DNS) of particle-laden turbulent flows have shown that singleparticle statistics, such as the turbulent dispersion of particles, is not significantly affected by the subgrid-scale (SGS) motions of LES, except near the wall, as they are mainly controlled by large-scale eddies, but particle-pair statistics, such as particle collision and preferential concentration, are sensitive to small-scale eddies (Yeh and Lei 1991; Uijttewaal and Oliemans 1996; Wang and Squires 1996; Armenio et al. 1999; Yamamoto et al. 2001; Fede and Simonin 2006). Therefore, in the present LCM, the transport of a droplet is calculated by following the trajectory of a Lagrangian droplet using LES, while the collision/coalescence process and preferential concentration are parameterized.

\section{Simulation}

A two-dimensional rising warm air bubble is triggered by an initial potential temperature difference $\theta^{*}$ given by

$\theta^{*}=\theta_{0}^{*} \exp \left[-\frac{1}{2}\left(\left(\frac{y-y_{c}}{a_{y}}\right)^{2}+\left(\frac{z-z_{c}}{a_{z}}\right)^{2}\right)\right]$.

where $y_{c}=1,920 \mathrm{~m}$ and $z_{c}=170 \mathrm{~m}$ mark the center of the bubble, a_ $\{\mathrm{y}\}=200 \mathrm{~m}$ and $a_{z}=150 \mathrm{~m}$ the radius of the bubble, and $\theta_{0}^{*}=0.4 \mathrm{~K}$ the maximum temperature difference. It is homogeneous in the $x$-direction. The model domain is $1,280 \mathrm{~m} \times 3,840 \mathrm{~m} \times 3,840 \mathrm{~m}$ along the $x, y$, and $z$ directions with a grid spacing of $20 \mathrm{~m}$ in all directions. The periodic boundary condition is applied laterally, and no slip and free slip boundary conditions are applied at the bottom and the top, respectively.

The background condition of the simulations is based on the Rain in Cumulus over the Ocean (RICO) field study (Rauber et al. 2007). The initial profiles of potential temperature and specific humidity derived from RICO are shown in Fig. 1. These are the same profiles used in the LES intercomparison of the cloud layer in RICO (van Zanten et al. 2011) except that there is no background wind in the present simulation. RICO is a comprehensive field study of shallow cumulus convection that was located in the winter trade-winds of the northwestern Atlantic Ocean, just upwind of the Islands of Antigua and Barbuda, during December 162004 and January 8 2005. The mean value of area-averaged precipitation during the period was about $21 \mathrm{~W} \mathrm{~m}^{-2}$, which is just less than $1 \mathrm{~mm} \mathrm{day}^{-1}$.

Super-droplets are released at the beginning of the simulation and uniformly distributed all over the model domain, up to a height of $2,800 \mathrm{~m}$. The average distance between super-droplets is initially $4.5 \mathrm{~m}$, yielding a total number of roughly $1.5 \times 10^{8}$ and about 87 super-droplets per grid box. Using an initial weighting factor of $9 \times 10^{9}$, the droplet concentration of approximately $100 \mathrm{~cm}^{-3}$ is represented. Riechelmann et al. (2012) showed that a maximum time step size of $0.1 \mathrm{~s}$ and a minimum number of about 80 initial droplets per grid box are necessary for the convergence of solutions from the sensitivity tests of the present LCM. We presume that these criteria can still be applied to the present simulation, because the roles of condensation and collision are less important during precipitation.

\section{Results}

\subsection{Evolution of cloud motion}

Evolution of a convective cloud simulated by LCM is illustrated by vertical cross sections of the fluid vertical 
Fig. 1 Initial profiles of (a) potential temperature $\theta$ and (b) specific humidity $q$
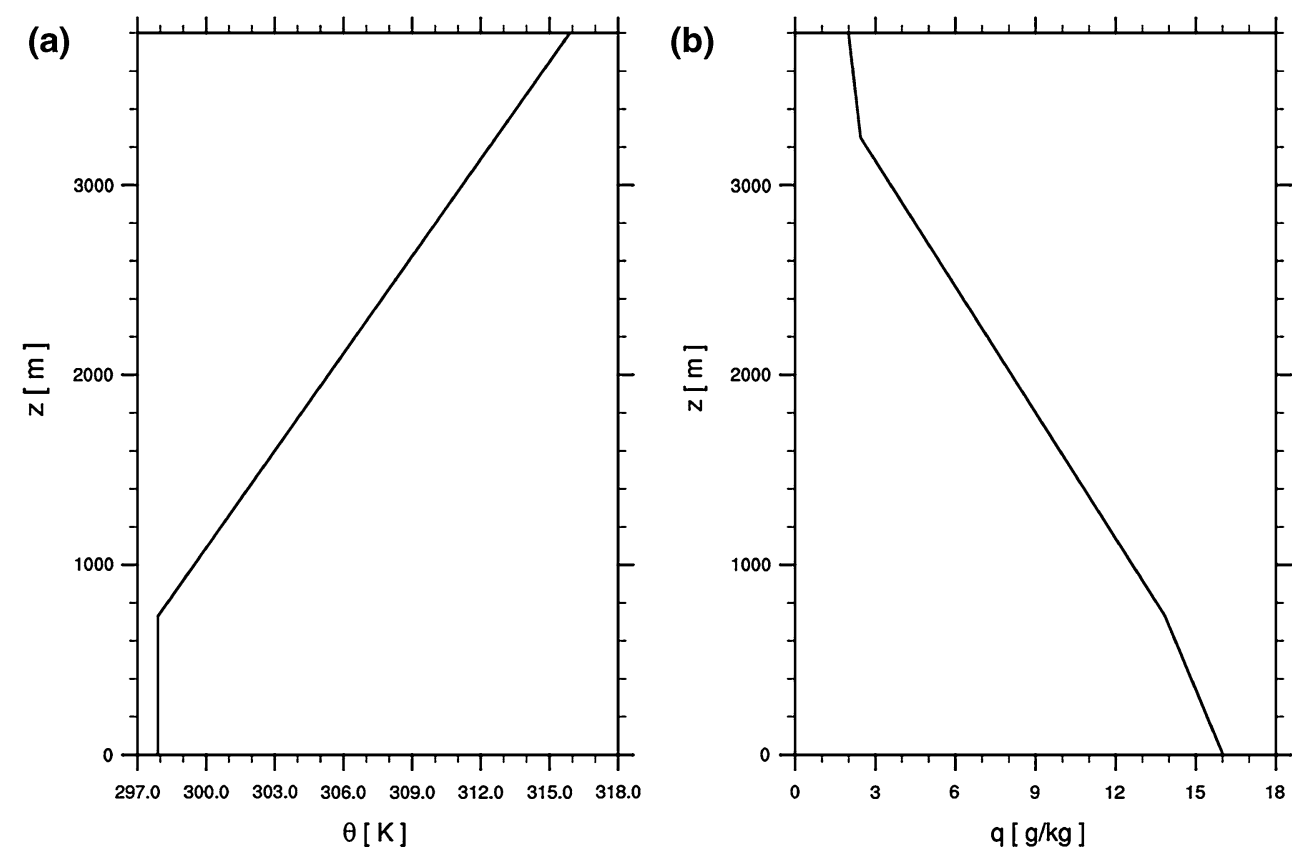

velocity $w$, liquid water mixing ratio $q_{l}$, and dissipation rate $\varepsilon$, averaged along the $x$-direction at $t=10,17,23$, and $30 \mathrm{~min}$, which correspond to the stages of the initial updraft, the fully developed convective cloud, the initiation of rain, and the active rainfall, respectively (Figs. 2, 3, 4). Here $\varepsilon$ was calculated from the parameterization of SGS turbulence in the LES model (Riechelmann et al. 2012). Vertical profiles of the corresponding variables at the center $(1,810 \mathrm{~m}<y$ $<2,010 \mathrm{~m}$ ) are shown together, while the results from Hall and AW kernels are compared.

The typical pattern of a rising single cloud appears at $t=10 \mathrm{~min}$, such as the dipole pattern of $w$ and the increase of its size through entrainment. The buoyancy force generates a well-developed turbulent thermal characterizing a convective cloud at $t=17 \mathrm{~min}$, with the maximum values of $q_{l}$ and $\varepsilon$ appearing near the cloud top. The values of $q_{l}$ and $\varepsilon$ are the largest at this stage. The initiation of precipitation at $t=23$ min induces downdraft, induced by the evaporation of droplets. Most properties of a convective cloud are not affected by the difference in the collection kernels, but the value of $q_{l}$ is slightly smaller within the cloud and slightly larger below the cloud at $t=23 \mathrm{~min}$ in the AW kernel, as a result of earlier and stronger precipitation. The maximum value of $\varepsilon$ is about $30 \mathrm{~cm}^{2} \mathrm{~s}^{-3}$, which is within the range $10-100 \mathrm{~cm}^{2} \mathrm{~s}^{-3}$ obtained for small cumulus clouds (MacPherson and Isaac 1977; Mazin et al. 1984; Siebert et al. 2006).

\subsection{Precipitation initiation}

Figure 5 shows the evolution of mass density distribution $g(\ln r)$ for both cases of Hall and AW kernels. Here $g(\ln r)$ is obtained using the mass of the super-droplets inside the cloud, multiplied by the respective weighting factor. The cloud is defined as the region where $q_{l}>1 \times 10^{-2} \mathrm{~g} / \mathrm{kg}$. Until $t=10 \mathrm{~min}$, the droplet growth is essentially due to condensation, and the difference between Hall and AW kernels does not appear yet. It shows the broadening of the droplet size distribution, indicating the inhomogeneous mixing due to the entrained dry air (Brenguier and Grabowski 1993; Kogan et al. 1995; Lasher-Trapp et al. 2005). Entrainment also provides small droplets from outside of the cloud.

On the other hand, the bimodal pattern of the droplet spectrum, attributed to the collisional growth, starts to appear at $t=17 \mathrm{~min}$, with the second peak of the spectrum near $r=100 \mu \mathrm{m}$. At this stage the droplet growth is significantly enhanced when the effect of turbulence is included in the collection kernel (i.e., the AW kernel) in agreement with the previous studies (Xue et al. 2008; Grabowski and Wang 2009; Seifert et al. 2010; Wyszogrodzki et al. 2013). At $t=30 \mathrm{~min}$ large droplets with $r>200 \mu \mathrm{m}$ disappear from the spectrum, because they fall out of the cloud as rain.

In order to clarify the droplet growth in detail, we investigate the distribution of the droplet spectra together with the distributions of liquid-water mixing ratio $\left(q_{l}\right)$ and fluid velocity $\left(u_{i}\right)$ (Fig. 6). During the condensation-dominated stage at $t=10 \mathrm{~min}$, the droplet spectra are unimodal everywhere. The droplet size distribution tends to be broader near the cloud top and, to a less degree, near the sides, which are the regions affected by the entrained dry air (Baker et al. 1980; Brenguier and Grabowski 1993; 
Fig. 2 Cross sections of fluid vertical velocity $w$ (left) and vertical profiles of its variance $w^{\prime 2}=(w-\bar{w})^{2}$ with $\bar{w}=0$ at the center $(1810 \mathrm{~m}<\mathrm{y}<2010 \mathrm{~m})$ (Hall: red; AW: blue) (right) at $t=10,17,23,30 \mathrm{~min}$. All values are averaged along the $x$-direction
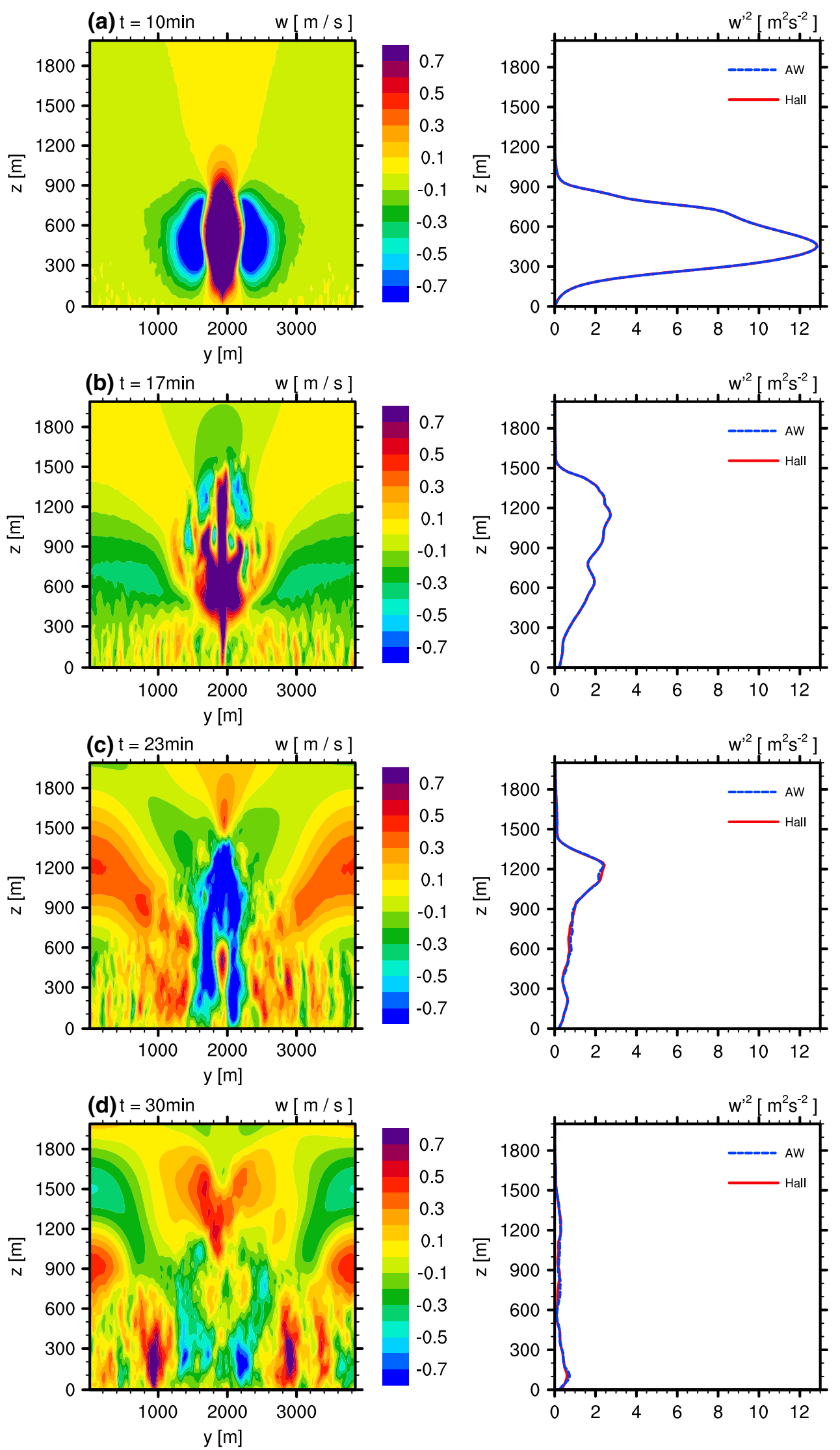
Fig. 3 Cross sections of liquid water mixing ratio $q_{l}$ (left) and vertical profiles of $q_{l}$ at the center (Hall: red; AW: blue) (right) at $t=10,17,23$,

$30 \mathrm{~min}$. All values are averaged along the $x$-direction
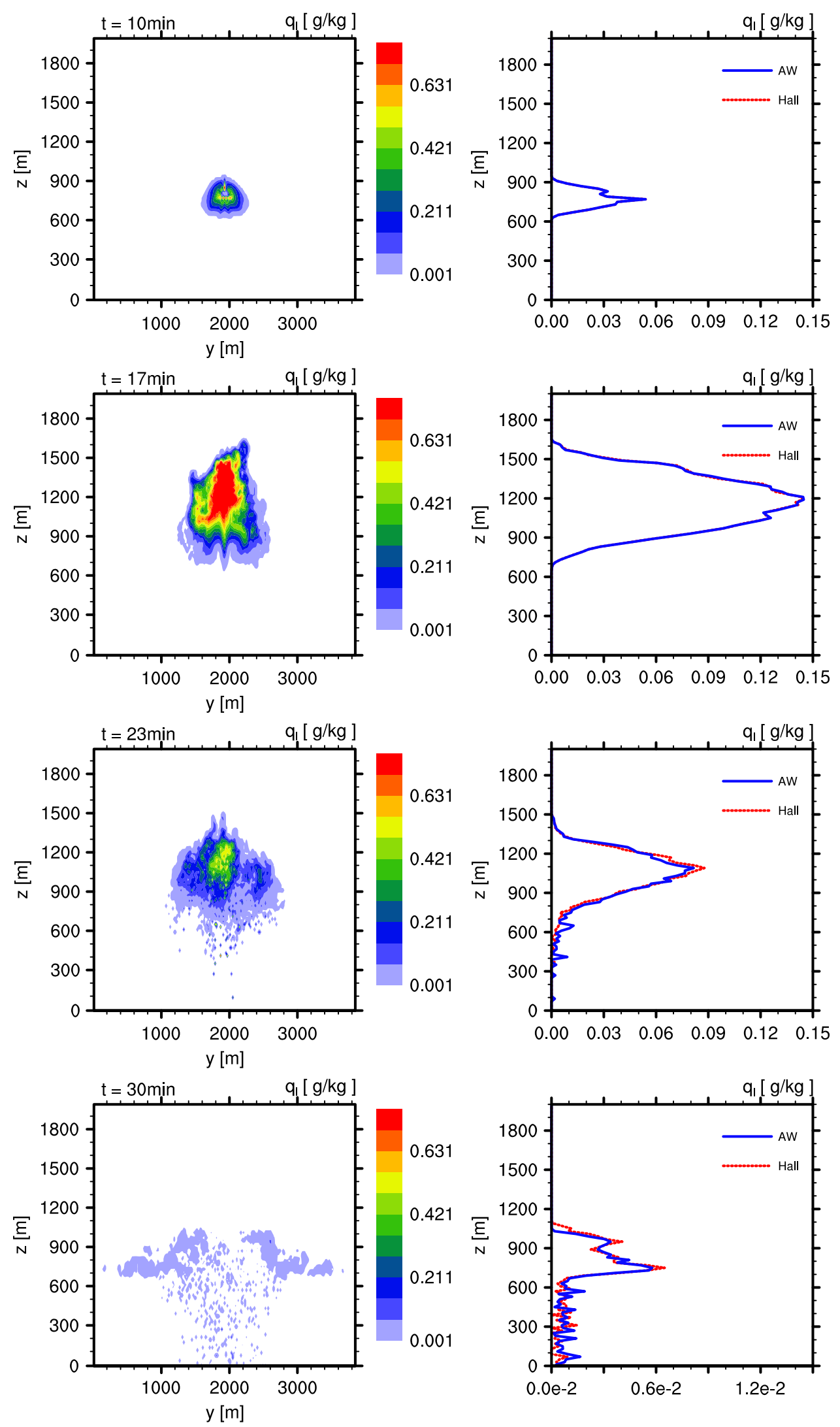
Fig. 4 Cross sections of dissipation rate $\varepsilon$ (left) and vertical profiles of $\varepsilon$ at the center (Hall: red; AW: blue) (right) at $t=10,17,23$,

$30 \mathrm{~min}$. All values are averaged along the $x$-direction
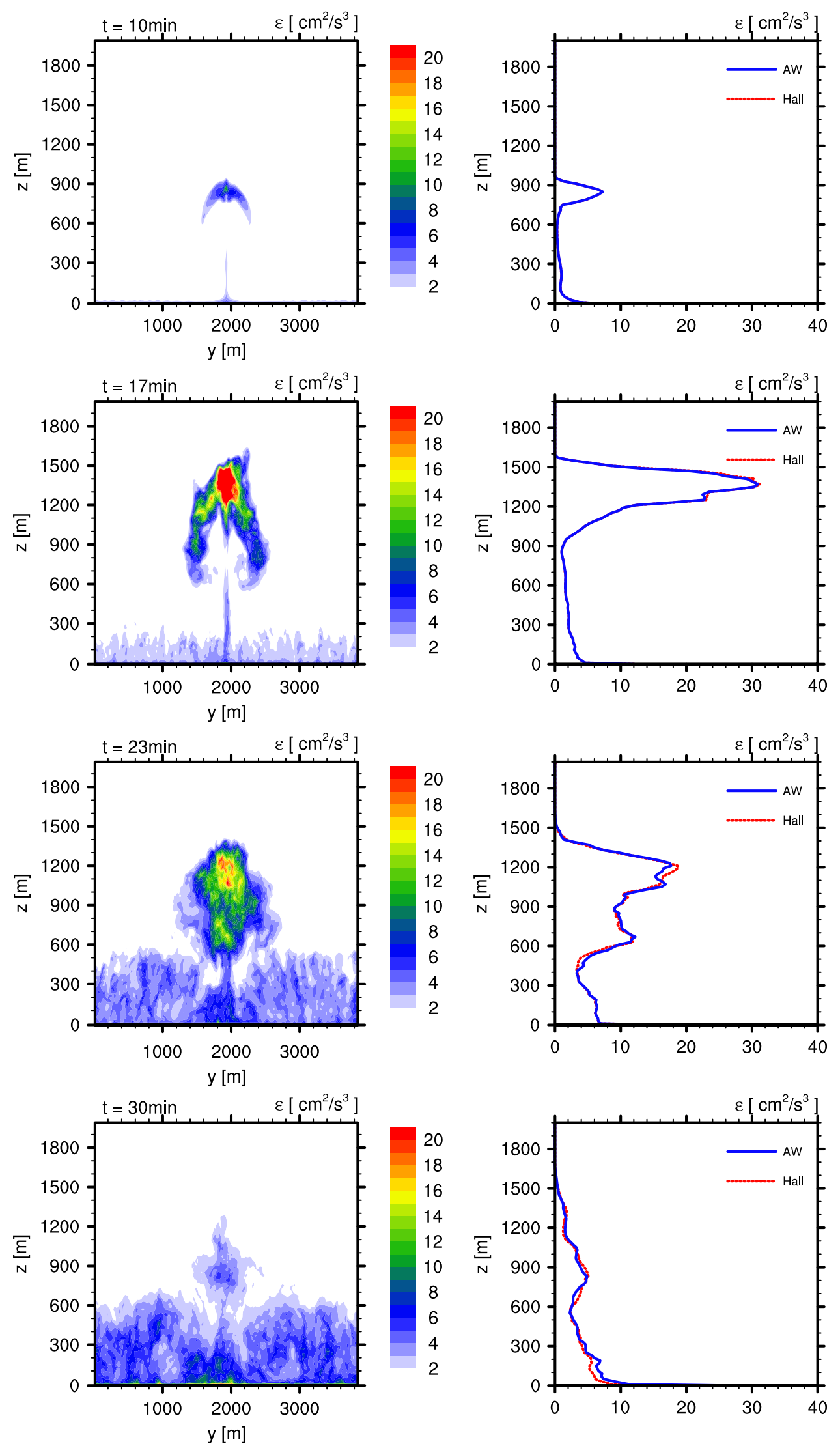

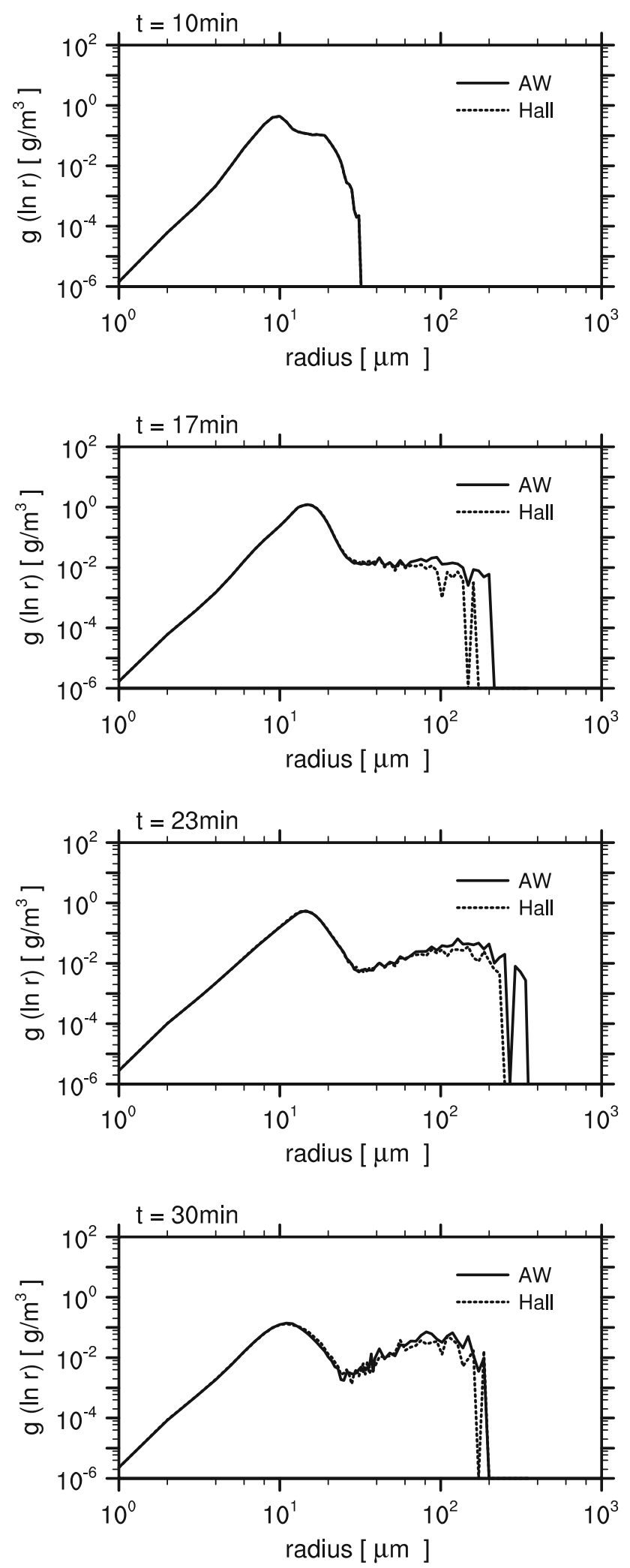

Fig. 5 Evolution of mass density distribution of droplets at $t=10$, 17, 23, 30 min (Hall: dashed, AW: solid)
Lasher-Trapp et al. 2005). At $t=17 \mathrm{~min}$, the droplet spectra in the lower part of the core still show the unimodal pattern, but the bimodal pattern is clearly identified near the cloud top and, to a less degree, near the sides, suggesting the formation of raindrops due to collision effects. With the start of rain at $t=23 \mathrm{~min}$, the spectral peak at the larger size in the lower part of the cloud is mainly attributed by sedimentation. As the direction of vertical velocity is reversed to downward, the droplet spectra near the cloud top become unimodal again and have a smaller mean size. As the rain progresses at $t=30 \mathrm{~min}$, the bimodal pattern within the cloud tends to disappear, since most raindrops fall out of the cloud.

The formation of raindrops near the cloud top, shown in Fig. 6, can be more clearly identified by locating the appearance of raindrops directly from the LCM results. In the present paper we regard a raindrop as a droplet with $r>40 \mu \mathrm{m}$. Figure 7 shows the evolution of instantaneous distributions of super-droplets in the vertical cross section with the band thickness of $100 \mathrm{~m}(500<x<600 \mathrm{~m})$ during $t=15-23 \mathrm{~min}$. Initially raindrops only appear near the cloud top and in the downdraft region away from the center $(t=15,17,19 \mathrm{~min})$. Subsequently, raindrops settle gravitationally and are dispersed within a cloud by turbulent mixing, before falling out of the cloud $(t=21$, $23 \mathrm{~min})$. A remarkable agreement is found between the pattern of precipitation initiation shown in Fig. 7 and the observation during RICO by Small and Chuang (2008). They found that raindrops are found preferentially at the cloud top and in the downdraft region and tend to cluster with each other with the length scale about $100 \mathrm{~m}$. The appearance of raindrops within $20 \mathrm{~min}$ in the present simulation is also consistent with the typical time scale of warm rain formation (e.g., Rogers and Yau 1989).

The critical role of entrainment and mixing in the formation of raindrops in a cumulus cloud has been proposed in many previous studies (Cooper et al. 2013; Lasher-Trapp et al. 2005; Brenguier and Grabowski 1993; Baker et al. 1980). Entrainment of unsaturated air from the environment results in the additional activation of entrained $\mathrm{CCN}$, providing a source of small cloud droplets. Entrainment also generates strong temperature and moisture fluctuations near the cloud edge and thus causes droplets to experience different integral supersaturations over time. Furthermore, the enhanced turbulence, caused by evaporative cooling due to the entrained unsaturated air, helps enhance the collision efficiency. These processes promote broadening the droplet size distribution and thus produce raindrops. On the other hand, entrainment can be detrimental to raindrop formation by reducing LWC. 
(a) $\mathrm{t}=10 \mathrm{~min}$
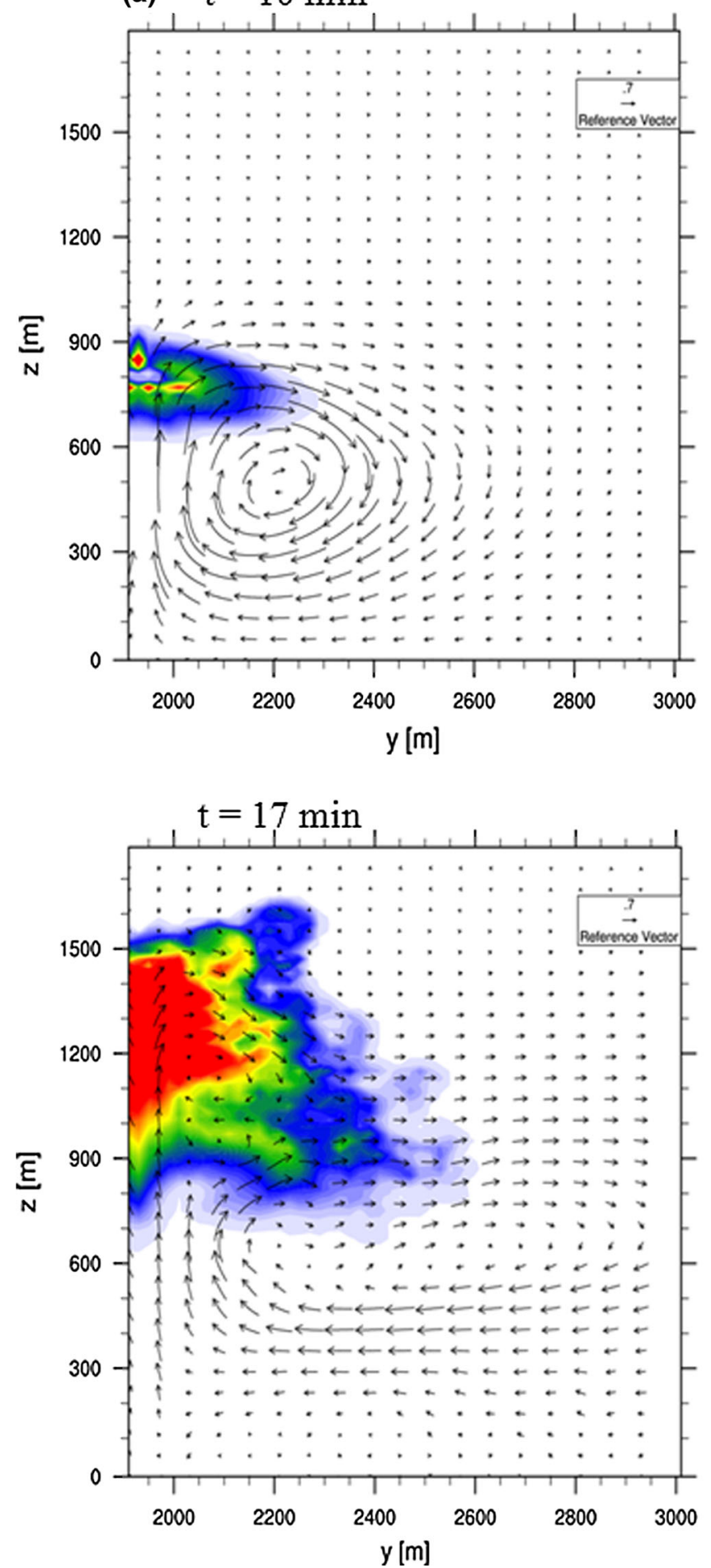

(b)

0.7

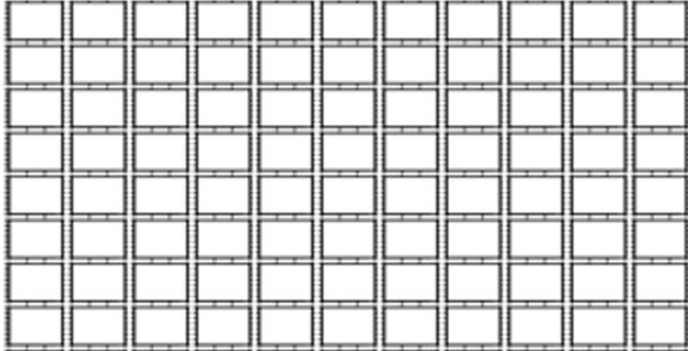

0.401

0.301

7.

$\Lambda$ त $\Lambda$

0.201

14

0.101

0.001

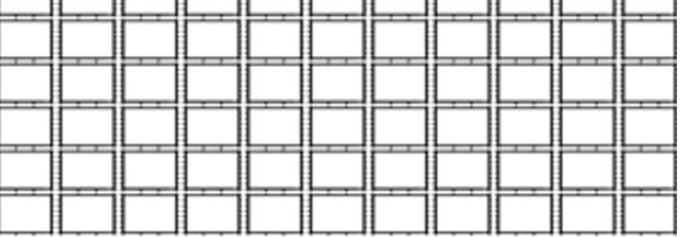

0.7

0.601

0.501

0.401

0.301

0.301

0.201

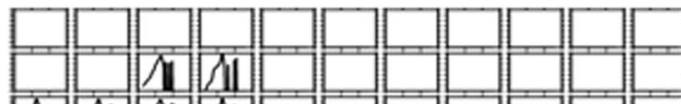

19M

M $1 M$

1.14 14

1. 1 M

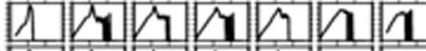

1. 1 M 1

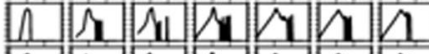

nh 1 an

h h h r

$1 . \square 1$

0.101

0.001

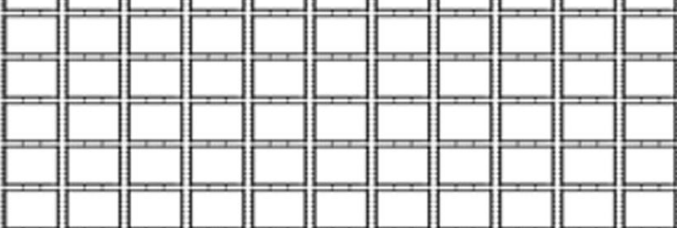

Fig. 6 Distributions of the liquid-water mixing ratio $q_{l}(\mathrm{~g} / \mathrm{kg})$ overlapped with the fluid velocity vector $(\mathrm{m} / \mathrm{s})(l e f t)$ and the mass density distributions of droplets (right). Each box corresponds in scale and coordinates to those of Fig. 5 ( $t=10,17,23,30 \mathrm{~min})$

In order to understand why raindrops are generated in the entrainment zone near the cloud top, we examined the relevant physical conditions mentioned above. The distributions of zonal mean values, shown in Figs. 3 and 4, indicate that $q_{l}$ and $\varepsilon$ are smaller in the entrainment zone near the edge of a cloud than in the cloud core, although the maximum values appear near the cloud top. However, the instantaneous distributions of $q_{l}$ and $\varepsilon$ in the horizontal 


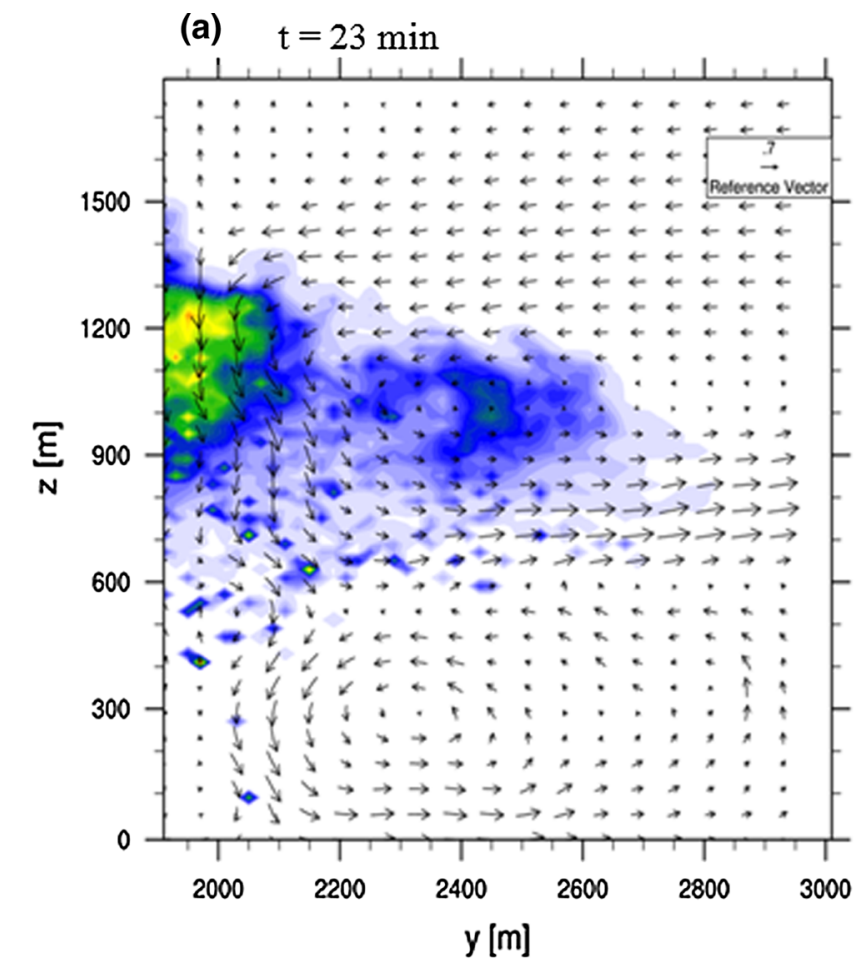

(b)
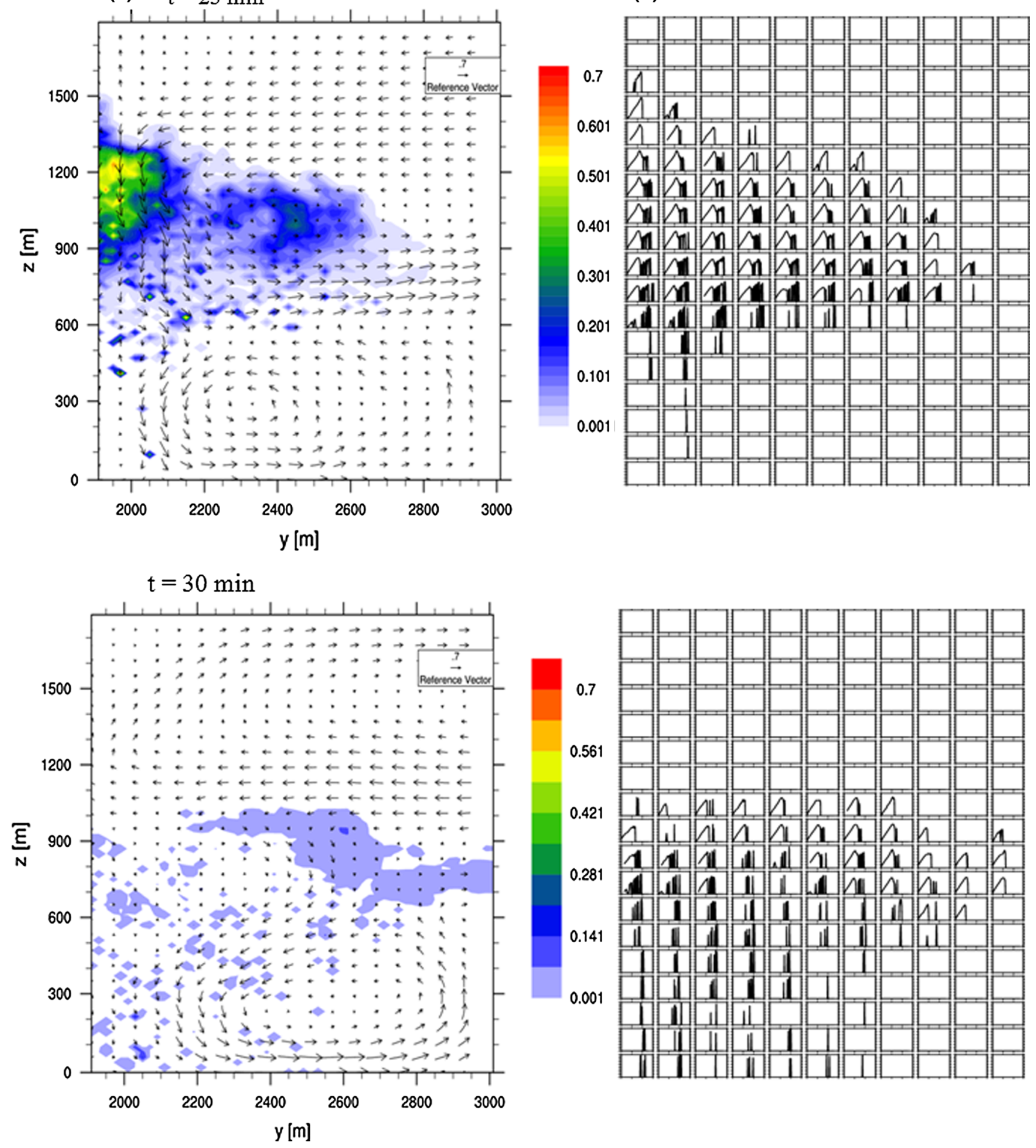

Fig. 6 continued

cross section at $t=17 \min (z=1,100,1,300 \mathrm{~m})$ reveals strong fluctuation of these values, demonstrating that the entrained air remains largely unmixed with the cloud air (Fig. 8). The values of $q_{l}$ within cloud parts in the entrainment zone away from the center at $z=1,300 \mathrm{~m}$ are actually larger than those in the cloud core at $z=1,100 \mathrm{~m}$, contrary to the zonal mean values shown in Fig. 3. Figure 8 also supports the fact that the values of $\varepsilon$ are highly fluctuating within a cloud up to a factor of 50 (Siebert et al. 2006). 


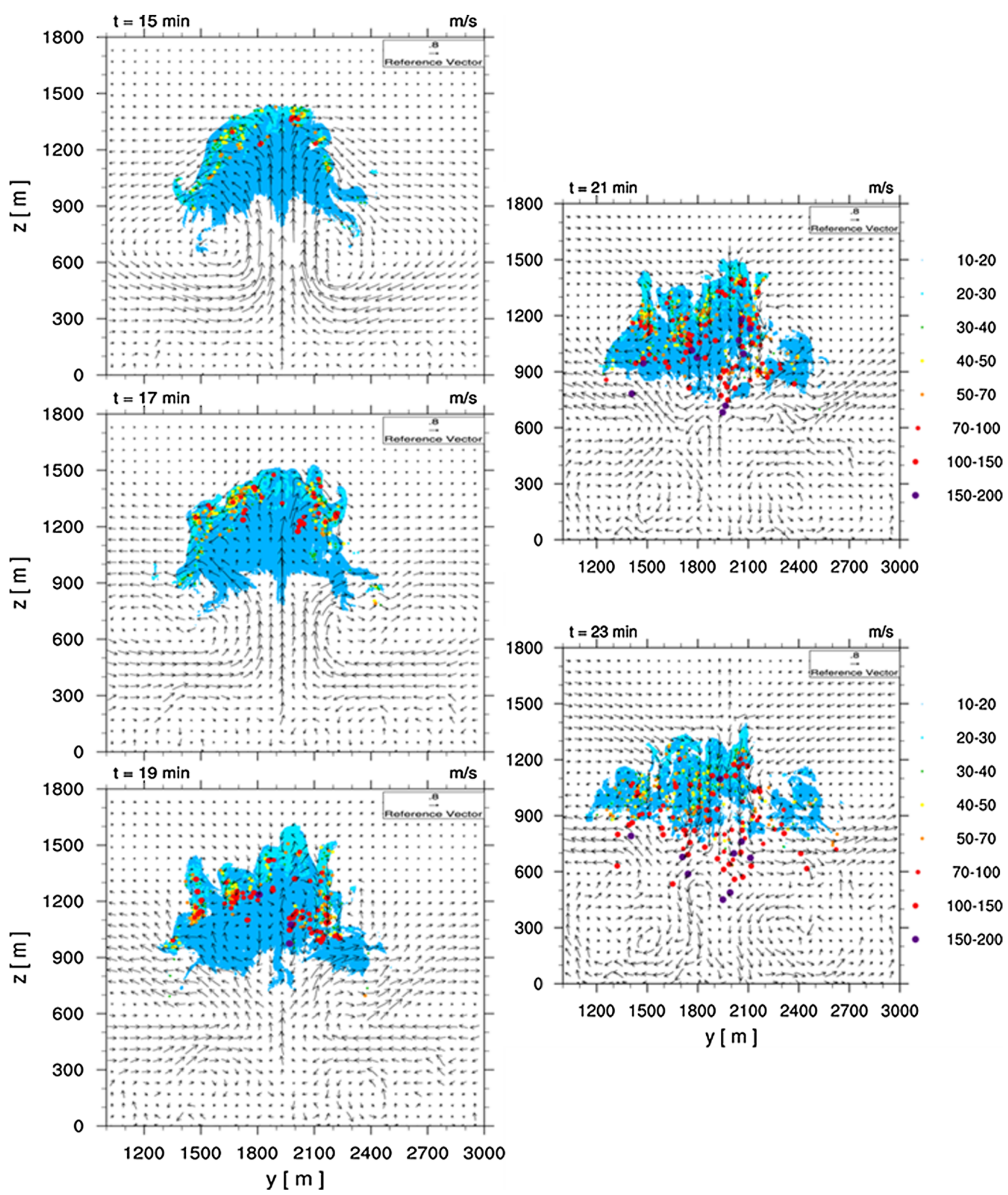

Fig. 7 Super-droplet distribution at the vertical cross section with the band thickness $100 \mathrm{~m}(500 \mathrm{~m}<x<600 \mathrm{~m})$, Vertical velocity field is overlapped for the AW case. Super-droplets are shown in different colors depending on its size $(t=15,17,19,21,23 \mathrm{~min})$

Figure 9 clearly identifies that not only the mean values but also the variance of $q_{l}$ and $\varepsilon$ are larger in the entrainment zone than in the cloud core. Here the cloud core represents the central band of $100 \mathrm{~m}$ at $z=1,100 \mathrm{~m}$, and the entrainment zone represents the region away from the central band at $z=1,300 \mathrm{~m}$, in the horizontal cross sections shown in 

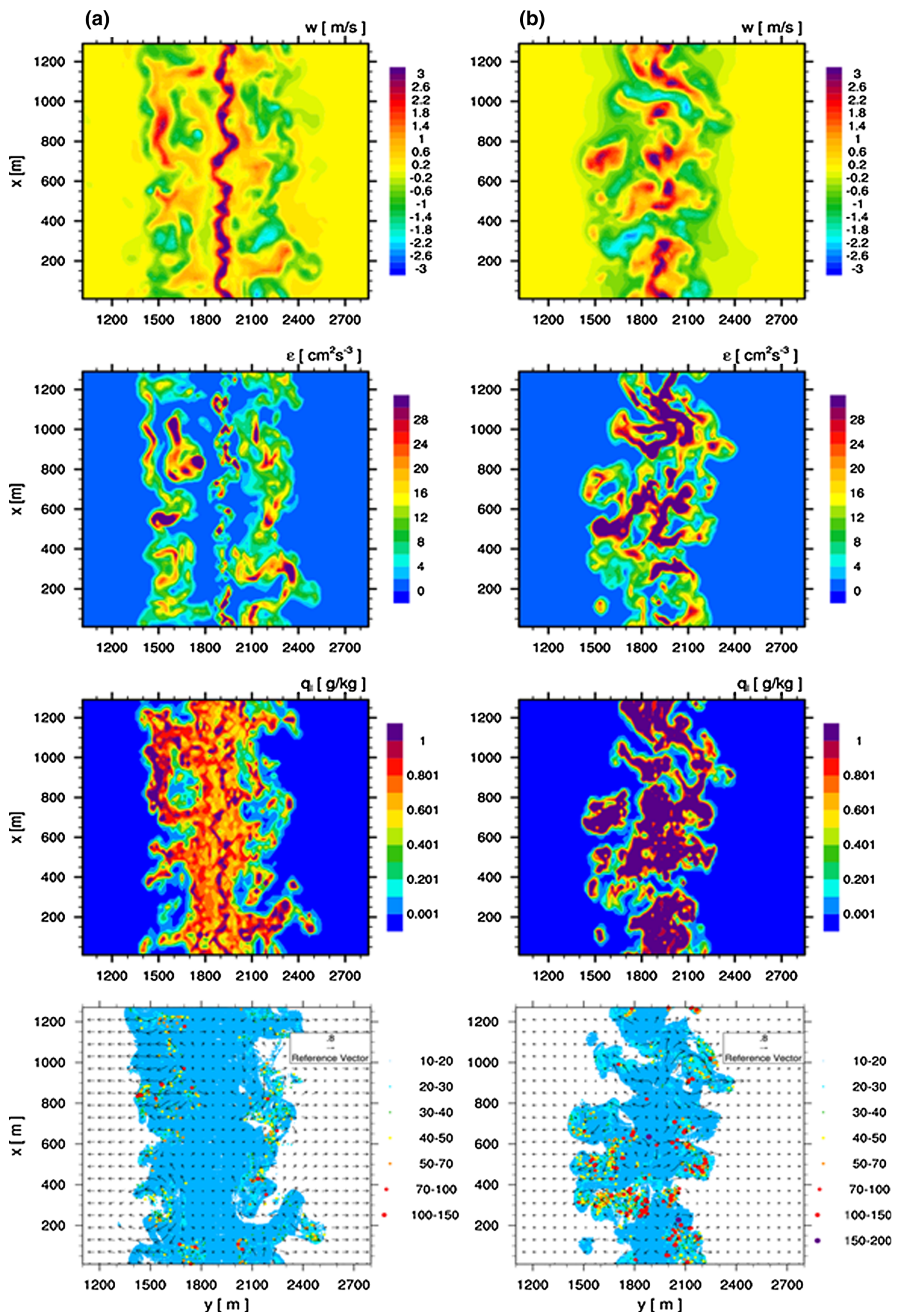
4Fig. 8 Distributions of vertical velocity $w$, liquid water mixing ratio $q_{l}$, and dissipation rate $\varepsilon$ at the horizontal cross section at $t=17 \mathrm{~min}$. At bottom are the distributions of super-droplets within the vertical thickness $100 \mathrm{~m}$ : a $z=1,100 \mathrm{~m}, \mathbf{b} z=1,300 \mathrm{~m}$

Fig. 8, and statistics are obtained only from the cloud region. Note that the droplet size distribution is much broader in the entrainment zone than in the cloud core before and during the raindrop formation ( $t=10$ and $17 \mathrm{~min}$ ) (Fig. 6). It implies that all the conditions favorable for the formation of raindrops, such as large $q_{l}$ and $\varepsilon$ and the broad droplet size distribution, are found in cloud parts in the entrainment zone near the cloud top.

The distribution of super-droplets at the same horizontal cross section, over the vertical thickness of $100 \mathrm{~m}$, reveals that raindrops tend to appear in the regions with strong entrainment, indicated by large velocity, and often in the regions with large $q_{l}$ and $\varepsilon$ (Fig. 8). Furthermore, it is interesting to observe that raindrops tend to cluster with each other, in agreement with the observation by Small and Chuang (2008). Finally, it is necessary to mention that raindrops appear about the same time at the cloud top, regardless of the collection kernel, although they are generated in a larger amount and grow faster with the AW kernel, as reflected in Fig. 5.

Contrary to the Eulerian cloud model, each droplet moves differently from each other and from the surrounding fluid, and the condensational growth and evaporation of each droplet are computed using the values of supersaturation interpolated to the position of a droplet within a grid cell. Therefore, each droplet experiences different integral supersaturations over time naturally in the LCM, and it leads to the broadening of droplet spectrum. For example, Cooper et al. (2013) and Lasher-Trapp et al. (2005) showed the broadening of droplet size distribution by calculating a microphysical parcel model that is run along trajectories produced by a standard cloud model. Meanwhile, in the entrainment zone, turbulence is generated by evaporative cooling, thus enhancing the collision efficiency. On the other hand, the cloud air remains largely unmixed with the entrained air in the entrainment zone, thus preventing $q_{l}$ from decreasing substantially.
Fig. 9 Relative frequency distributions of $q_{l}$ and $\varepsilon$ within the cloud region: a cloud core $(1,860 \mathrm{~m}<y<1,980 \mathrm{~m}$, $z=1,100 \mathrm{~m}), \mathbf{b}$ entrainment zone $(y<1,860 \mathrm{~m}$ or $y>1,980 \mathrm{~m}, z=1,300 \mathrm{~m})$ (a)
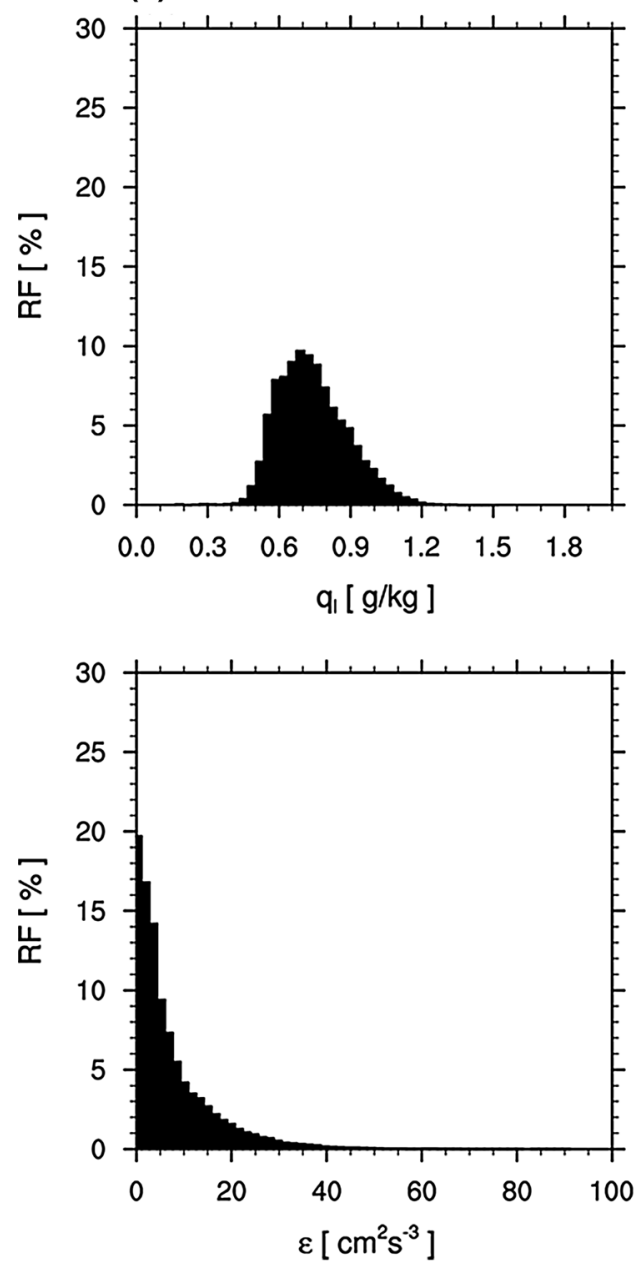

(b)
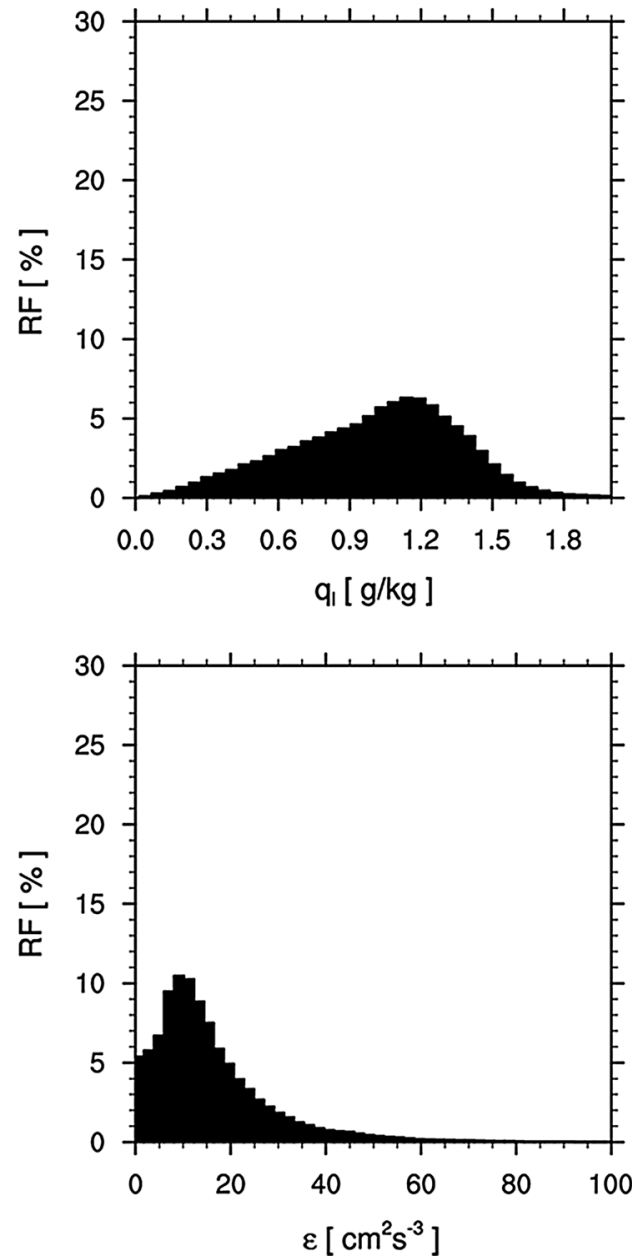


\subsection{Examination of microphysics parameterization}

In previous sections the LCM is shown to reproduce realistically the evolution of a precipitating cloud. Meanwhile, LCM can provide us the information on the motion of individual droplets directly, contrary to bulk and spectral bin models.

Most bulk models are developed based on the assumptions of the bimodal distribution of droplets, dividing into cloud droplets and raindrops according to the size (Kessler 1969; Emanuel 1994; Stensrud 2007). Cloud drops, whose radius is smaller than a critical radius $r_{c}$, are assumed to follow the fluid velocity, while the settling velocity is neglected. On the other hand, raindrops, whose radius is larger than $r_{c}$, are assumed to fall with the terminal velocity while being advected by the fluid velocity; i.e.,

$W=-w+w_{\mathrm{s}}$,

or $W=w_{\mathrm{s}}$ if $|w|<<w_{s}$. Note that (5) is obtained, when $\tau_{p}$ is much smaller than the time scale of a turbulent flow in

(a)
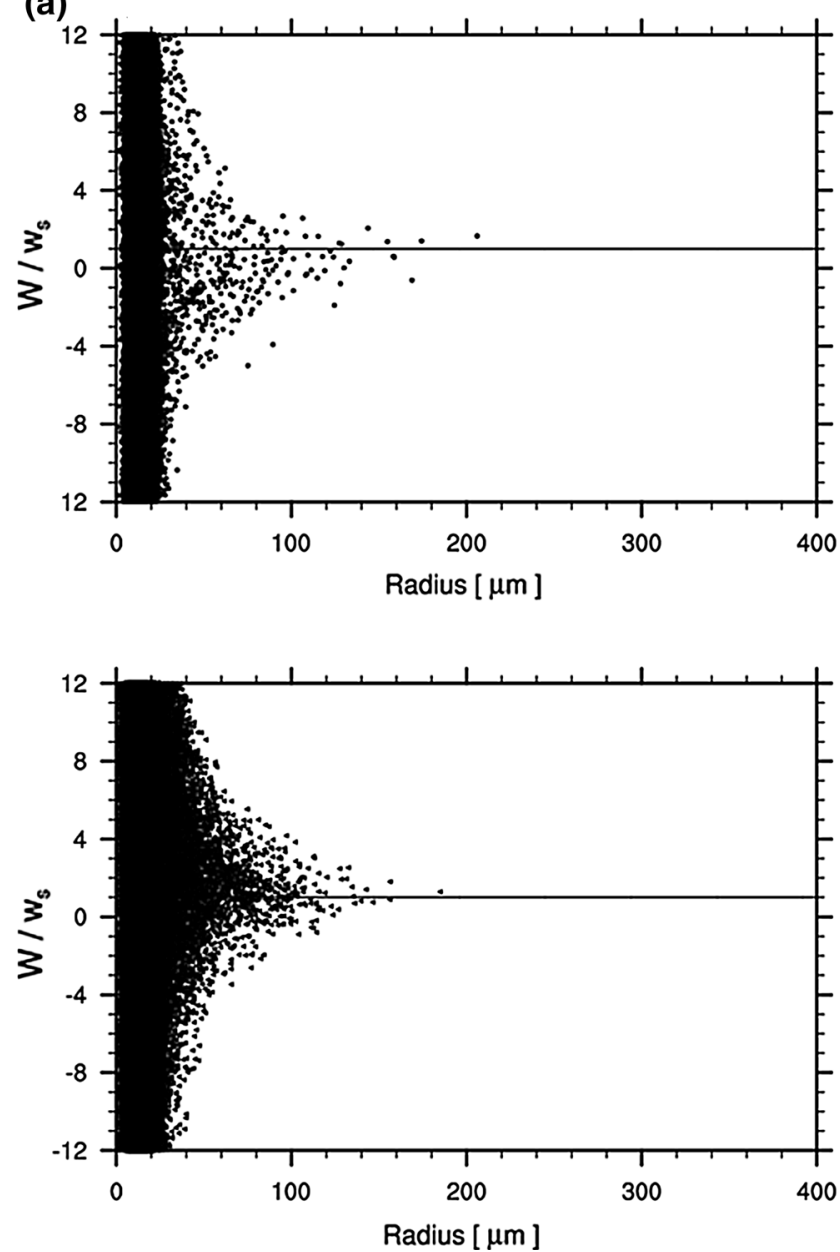

(2). In Sect. 4.2, $r_{c}=40 \mu \mathrm{m}$ is used, but a wide range of values is used in bulk models $\left(r_{c}=40-100 \mu \mathrm{m}\right)$ (Kessler 1969; Shiino 1983; Beheng and Doms 1986; Emanuel 1994; Seifert and Beheng 2001).

Table 1 Values of the rms of $\left(W-w_{\mathrm{s}}\right) / w_{\mathrm{s}}\left(=\sigma_{W / w_{\mathrm{s}}}\right)$ in the inner $(1,800 \mathrm{~m} \leq y<2,000 \mathrm{~m}, \quad$ at $t=17 \mathrm{~min}, \quad$ and $1,600 \mathrm{~m} \leq y<$ $2,200 \mathrm{~m}$, at $t=23 \mathrm{~min}$ ) and outer region of cloud droplets for $r=10,40,100 \mu \mathrm{m}(t=17,23 \mathrm{~min})$

\begin{tabular}{lrrrrr}
\hline Radius $(\mu \mathrm{m})$ & \multicolumn{2}{l}{$t=17 \mathrm{~min}$} & & \multicolumn{2}{c}{$t=23 \mathrm{~min}$} \\
\cline { 2 - 3 } \cline { 5 - 6 } & Inner & Outer & & Inner & Outer \\
\hline 10 & 73.14 & 42.35 & & 68.90 & 35.00 \\
40 & 4.55 & 4.10 & & 6.63 & 3.38 \\
100 & 1.32 & 0.90 & & 1.29 & 0.44
\end{tabular}

Here the thickness of the spectral band for the calculation is given proportional to $r$, i.e., $\Delta r=0.1 r(=1,4,10 \mu \mathrm{m})$
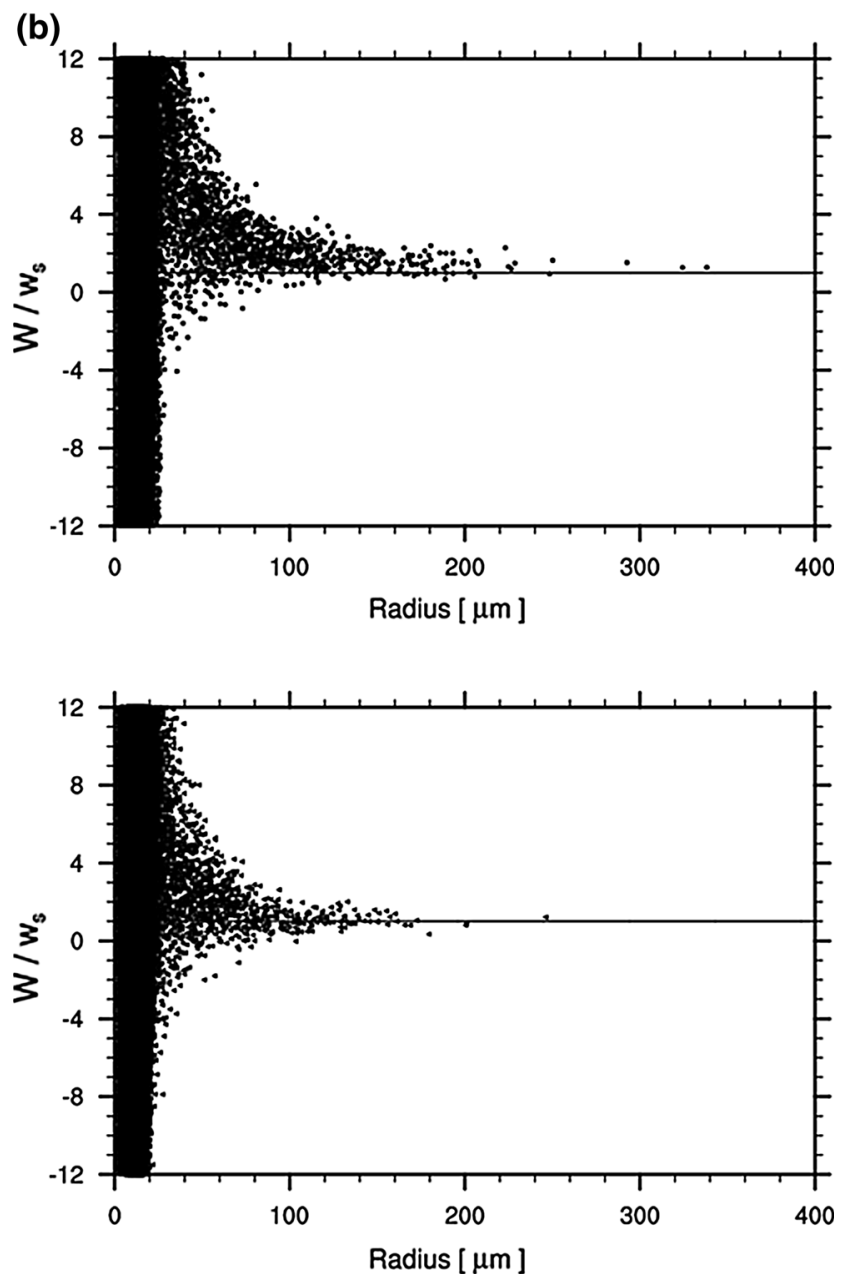

Fig. 10 Scatter plots of $W / w_{\mathrm{s}}$ in the inner (upper) $(1,800 \mathrm{~m} \leq y<2,000 \mathrm{~m}$, at $t=17 \mathrm{~min}$ and $1,600 \mathrm{~m} \leq y<2,200 \mathrm{~m}$, at $t=23 \mathrm{~min})$ and outer (lower) region of cloud droplets: a $t=17 \min ($ left), $\mathbf{b} t=23 \min$ (right) 
Bulk models are then made of equations to calculate the mixing ratios of cloud water and rainwater, $q_{C}$ and $q_{R}$ $\left(q_{l}=q_{C}+q_{R}\right)$. For example, as the simplest representation of warm cloud microphysics, Kessler (1969) suggested a bulk model as

$$
\frac{\mathrm{d} q_{C}}{\mathrm{~d} t}=\Phi-A-C+D_{C}
$$

$\frac{\mathrm{d} q_{R}}{\mathrm{~d} t}=-\frac{1}{\rho_{0}} \frac{\partial \Pi}{\partial z}+A+C-E+D_{R}$

Fig. 11 Relative frequency distribution of $\Delta W(=w-W)$ with the terminal velocity $w_{\mathrm{s}}$ (vertical line) for $r=10,40$, $100 \mu \mathrm{m}:$ a $t=17 \mathrm{~min}$,

b $t=23 \mathrm{~min}$ (a)
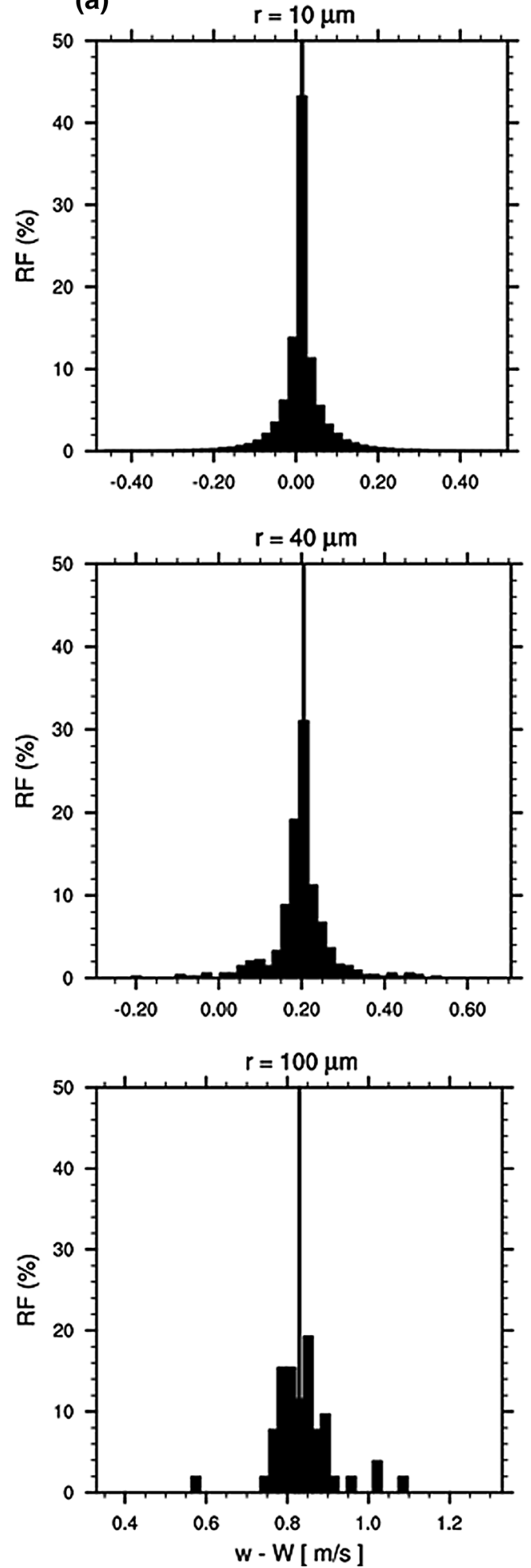

(b)
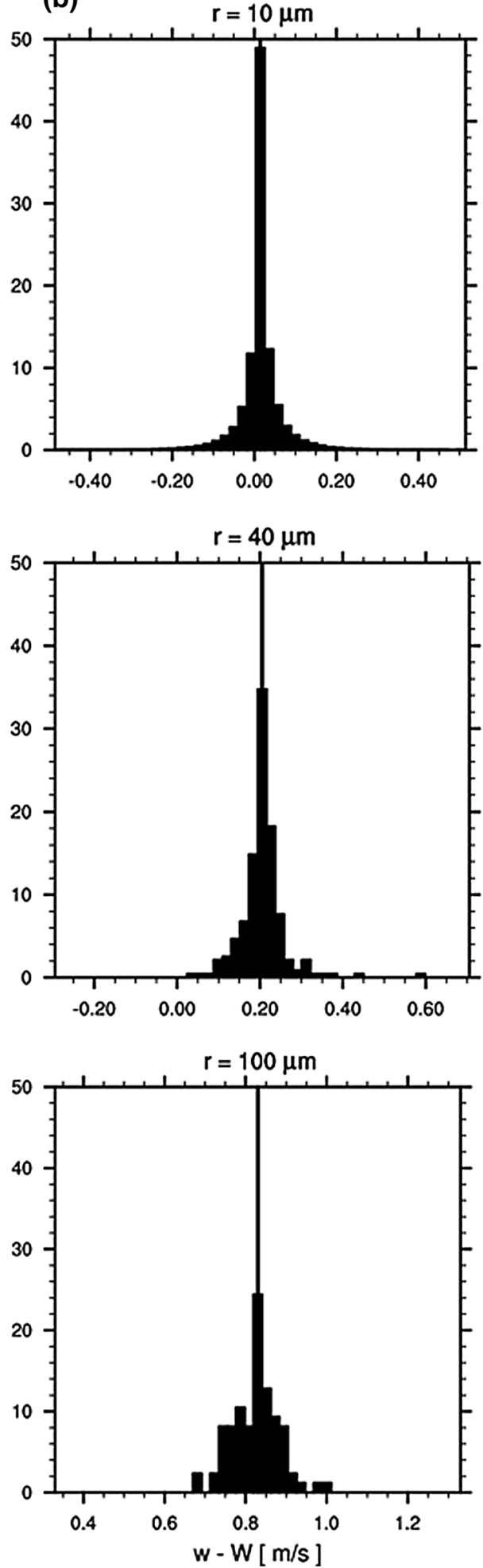
where $\Phi$ represents the contribution from condensation, $\Pi$ the precipitation flux, $A$ the autoconversion from cloud water to rainwater, $C$ the collection of cloud water by rainwater, $E$ evaporation, and $D_{C}$ and $D_{R}$ the turbulent diffusion of cloud water and rainwater, respectively.

In many bulk models, including Kessler (1969), the raindrop size distribution is assumed to follow the Marshall-Palmer distribution (Marshall and Palmer 1948) as

$n(r)=n_{0} \mathrm{e}^{-\lambda r}$

where $n_{0}$ and $\lambda$ are constants.

It is important to recognize that LCM can provide information for the parameterization of most terms in the RHS of (6) and (7) in principle. For example, $\Pi, D_{C}$ and $D_{R}$ can be evaluated by analyzing the transport of droplets, and $\Phi, C$ and $E$ can be evaluated by analyzing the corresponding contributions to droplets. Meanwhile, $A$ can be evaluated by analyzing the evolution of droplet spectrum as in spectral bin models.

With an aim to show the applicability of the LCM in the present work, however, we examine the following three hypotheses used in the Kessler's model (1969) by analyzing the LCM results. First, as to how the vertical velocity of a droplet varies with $r$ during the transition from a cloud drop to a raindrop. Second, how the raindrop size is dis-

Table 2 Values of the rms of $\Delta W-w_{\mathrm{s}}\left(=\sigma_{\Delta W}\right)$ and its ratio to $w_{\mathrm{s}}$ $\left(=\sigma_{\Delta W} / w_{\mathrm{s}}\right)$ for $r=10,40,100 \mu \mathrm{m}(t=17,23 \mathrm{~min})$

\begin{tabular}{llllll}
\hline Radius $(\mu \mathrm{m})$ & \multicolumn{2}{l}{$t=17 \mathrm{~min}$} & & \multicolumn{2}{l}{$t=23 \mathrm{~min}$} \\
\cline { 2 - 3 } \cline { 5 - 6 } & $\mathrm{rms}(\mathrm{cm} / \mathrm{s})$ & $\mathrm{rms} / w_{\mathrm{s}}$ & & $\mathrm{rms}(\mathrm{cm} / \mathrm{s})$ & $\mathrm{rms} / w_{\mathrm{s}}$ \\
\hline 10 & 6.92 & 4.69 & & 5.55 & 3.76 \\
40 & 7.33 & 0.36 & 5.28 & 0.26 \\
100 & 7.56 & 0.09 & & 5.85 & 0.08 \\
\hline
\end{tabular}

tributed and whether the Marshall-Palmer distribution (8) is observed. Finally, how accurately the precipitation flux based on $w_{s}$ predicts the real one based on $W$.

Figure 10 shows the scatter plots of $W / w_{\mathrm{s}}$ vs. $r$ at $t=17$ and $23 \mathrm{~min}$. The plots are made separately for the inner region $(1,800 \mathrm{~m} \leq y<2,000 \mathrm{~m}$, at $t=17 \mathrm{~min}$, and $1,600 \mathrm{~m} \leq y<2,200 \mathrm{~m}$, at $t=23 \mathrm{~min}$ ) and for the outer region. The asymmetry at $t=23 \mathrm{~min}$ is due to the presence of strong downdraft. The range of $W / w_{\mathrm{s}}$, which is very large for small droplets $(r<20 \mu \mathrm{m})$, decreases rapidly with $r$, converging to $W / w_{s}=1(r>100 \mu \mathrm{m})$. This implies that $W$ is basically determined by the fluid velocity $w$, independent of $w_{\mathrm{s}}$, for droplets with $r<20 \mu \mathrm{m}$, but $W$ is mainly controlled by $w_{\mathrm{s}}$ for droplets with $r>100 \mu \mathrm{m}$, although a large variation of $W$ comparable to $w_{\mathrm{s}}$ is still present. One can regard the former $(r<20 \mu \mathrm{m})$ as cloud droplets $(W=-w)$ and the latter $(r>100 \mu \mathrm{m})$ as raindrops $\left(W=-w+w_{\mathrm{s}}\right)$, according to Fig. 10. The transition from cloud droplets to raindrops occurs for smaller $r$ in the outer region, in which the fluid velocities, both mean and turbulent, are smaller. Further evidence is provided by the evaluation of the root mean square (rms) values of $\left(W-w_{\mathrm{s}}\right) / w_{\mathrm{s}}\left(=\sigma_{W / w_{\mathrm{s}}}\right)$ in the inner and outer regions, as shown in Table 1. Table 1 also reveals that the rms of $W$ is comparable to $w_{\mathrm{s}}$, even for $r=100 \mu \mathrm{m}$.

We can also examine how accurately (5) represents the real droplet velocity $W$. The relative frequency distribution of $\Delta W=w+W\left(=w-V_{3}\right)$ for droplets with different sizes $r=10,40$, and $100 \mu \mathrm{m}$ reveals that the variance of $\Delta W-w_{\mathrm{s}}, \sigma_{\Delta W}$, is not so sensitive to $r$, although it tends to increase weakly with $r$, suggesting the effect of particle inertia (Fig. 11; Table 2). On the other hand, $\sigma_{\Delta W} / w_{\mathrm{s}}$ decreases with $r$. These suggest that the deviation of $W$ from $w-w_{\mathrm{s}}$ is small in raindrops $(r=100 \mu \mathrm{m})$, but not negligible.
Fig. 12 The raindrop size distribution at $t=23 \mathrm{~min}$ (Hall: red; AW: blue). The solid line (orange) represents the Marshall-Palmer distribution with the precipitation rate $0.1 \mathrm{~mm} \mathrm{~h}^{-1}$

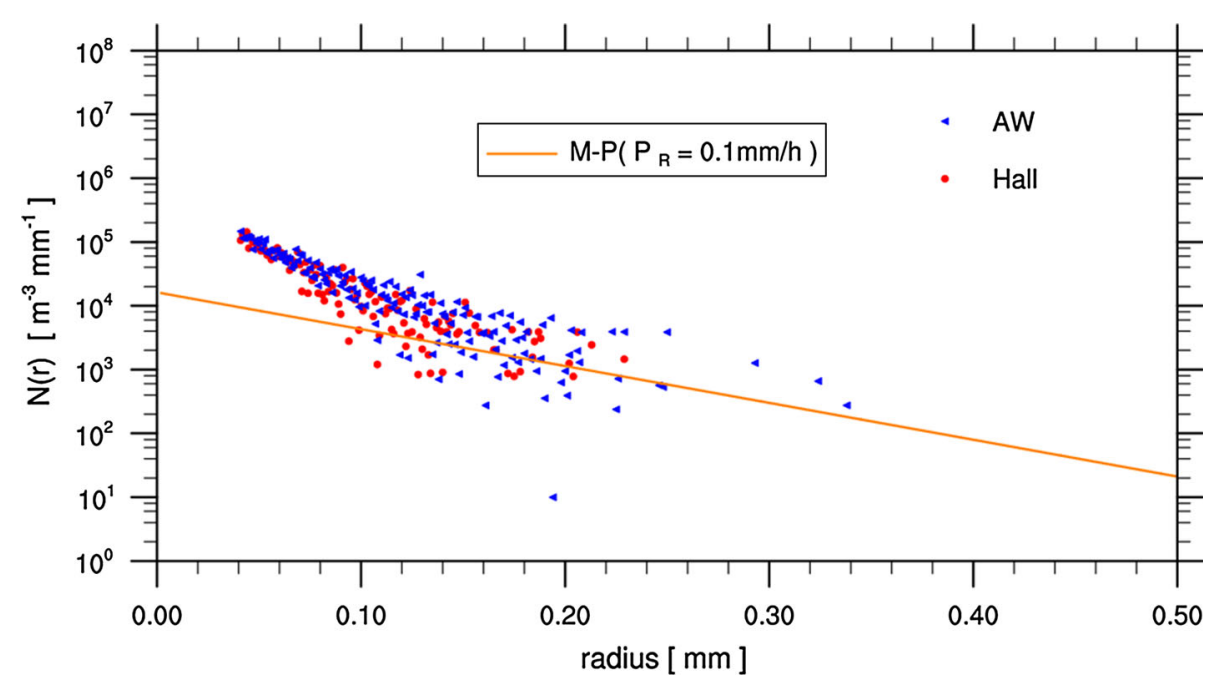


Although the transition from a cloud droplet $(W=-w)$ to raindrops $\left(W=-w+w_{\mathrm{s}}\right)$ with increasing $r$ is expected from (5), no information has been available so far on how $W$ varies with $r$ (Fig. 10) and what the probability distribution of $W$ is (Fig. 11; Tables 1 and 2). The present results show that they are affected by the turbulent flow field in a cloud as well as $r$.
Figure 12 reveals that the raindrop size distribution from LCM decreases exponentially with $r$, as in the MarshallPalmer distribution, given by (8). Here $n(r)$ is calculated by the average over the region with rain for droplets with $r>40 \mu \mathrm{m}$ at $t=23 \mathrm{~min}$. The variations of $n(r)$ with $r$ using the empirical constants, $n_{0}=8 \times 10^{-3} \mathrm{~m}^{-3} \mathrm{~mm}^{-1}$ and $\lambda=4.1 P_{R}^{-0.21} \mathrm{~mm}^{-1}$ from Marshall and Palmer (1948)
Fig. 13 The precipitation flux profile at $t=24,26,28 \mathrm{~min}$, using the actual fall velocity $W$ (left) and the terminal velocity $w_{\mathrm{s}}\left(\right.$ right): a $r_{c}=40 \mu \mathrm{m}$, b $r_{c}=100 \mu \mathrm{m}$ (a) $\mathrm{w}$
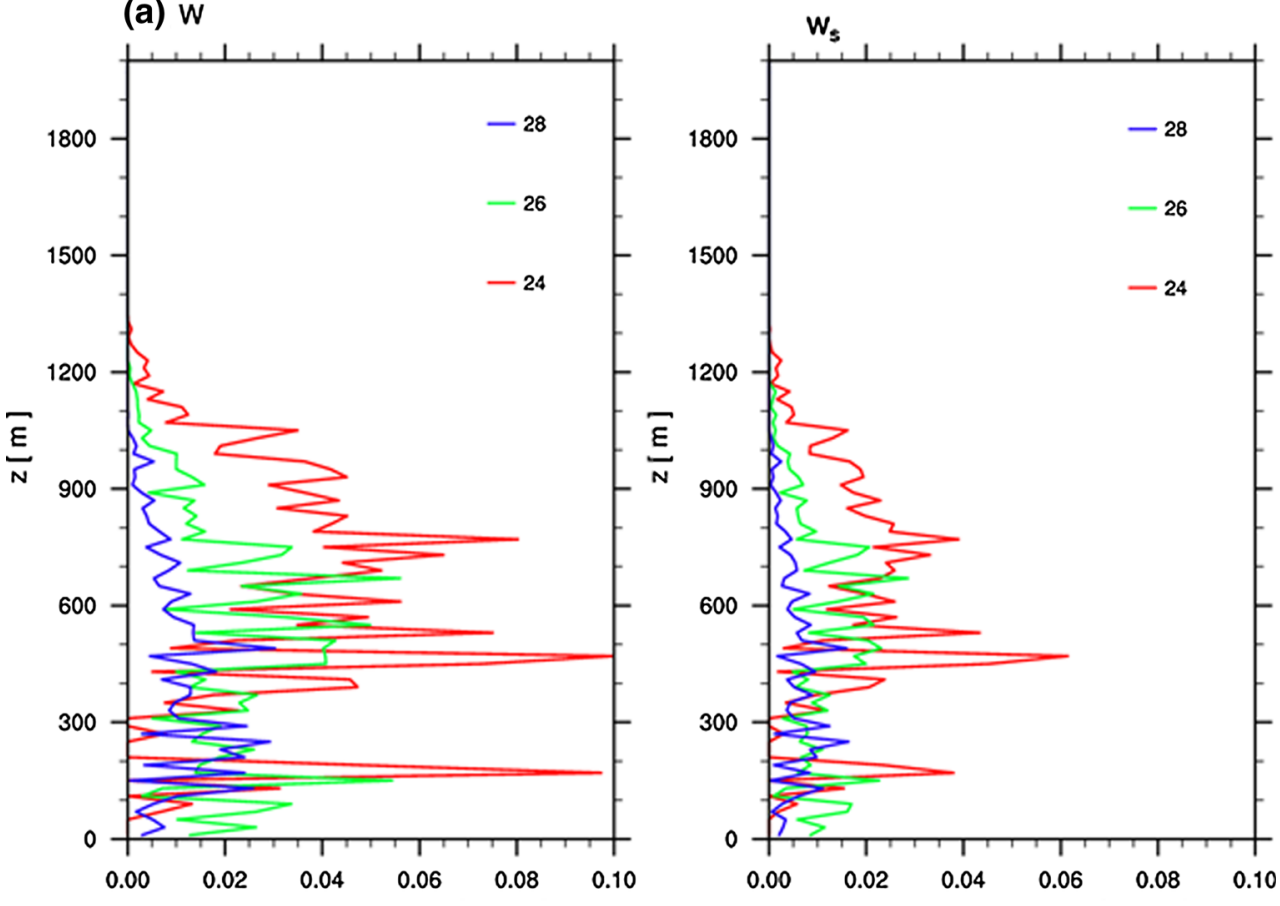

(b) $w$

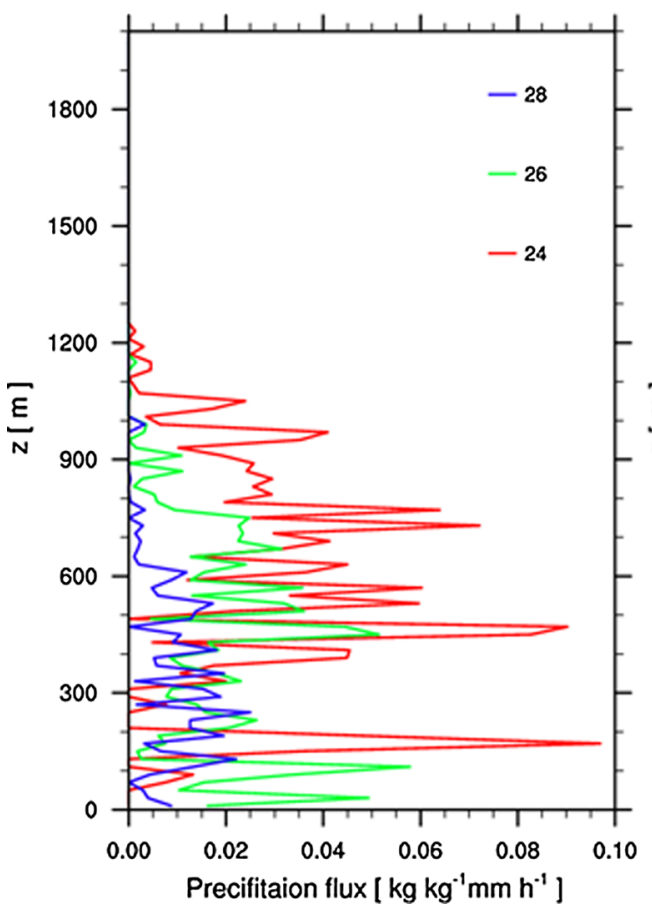

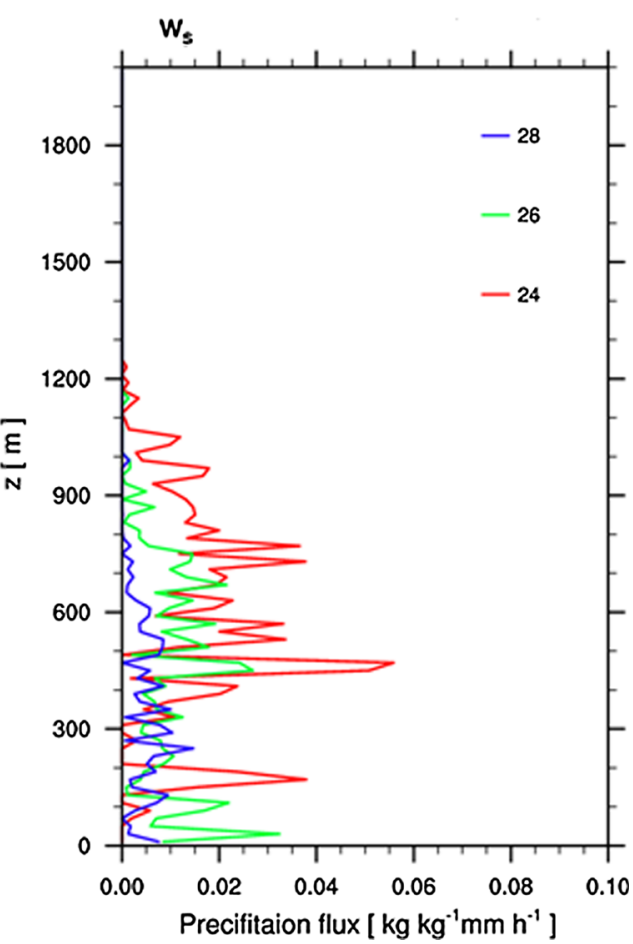


with $P_{R}=0.1 \mathrm{~mm} \mathrm{~h}^{-1}$ are also shown. It is important, however, to notice that the direct quantitative comparison of $n(r)$ is not appropriate here, because only the precipitation from a single cloud is considered. It may be also necessary to mention that the formula (8) is obtained mainly from the measurements of larger raindrops by Marshall and Palmer (1948) and that the breakup of droplets is not included in the present model. Nonetheless, it is worthwhile to mention that Fig. 12 is the first attempt to obtain the raindrop size distribution directly from simulation, taking advantage of LCM. The decrease of $n(r)$ with $r$ tends to be slightly slower in the case of the AW kernel, as expected from the higher precipitation rate.

In the LCM, $\Pi$ can be calculated directly using the downward velocities of individual raindrops $\left(r>r_{c}\right)$ as

$\Pi=\int_{r_{c}}^{\infty} W \frac{\rho_{1}}{\rho_{0}} \frac{4 \pi}{3} r^{3} n(r) \mathrm{d} r$,
Fig. 14 Evolution of the precipitation flux profiles at $t=20,22,24,26,28 \mathrm{~min}:$ a Hall kernel, b AW kernel
Fig. 15 Time series of the precipitation rate (dashed Hall kernel, solid AW kernel)
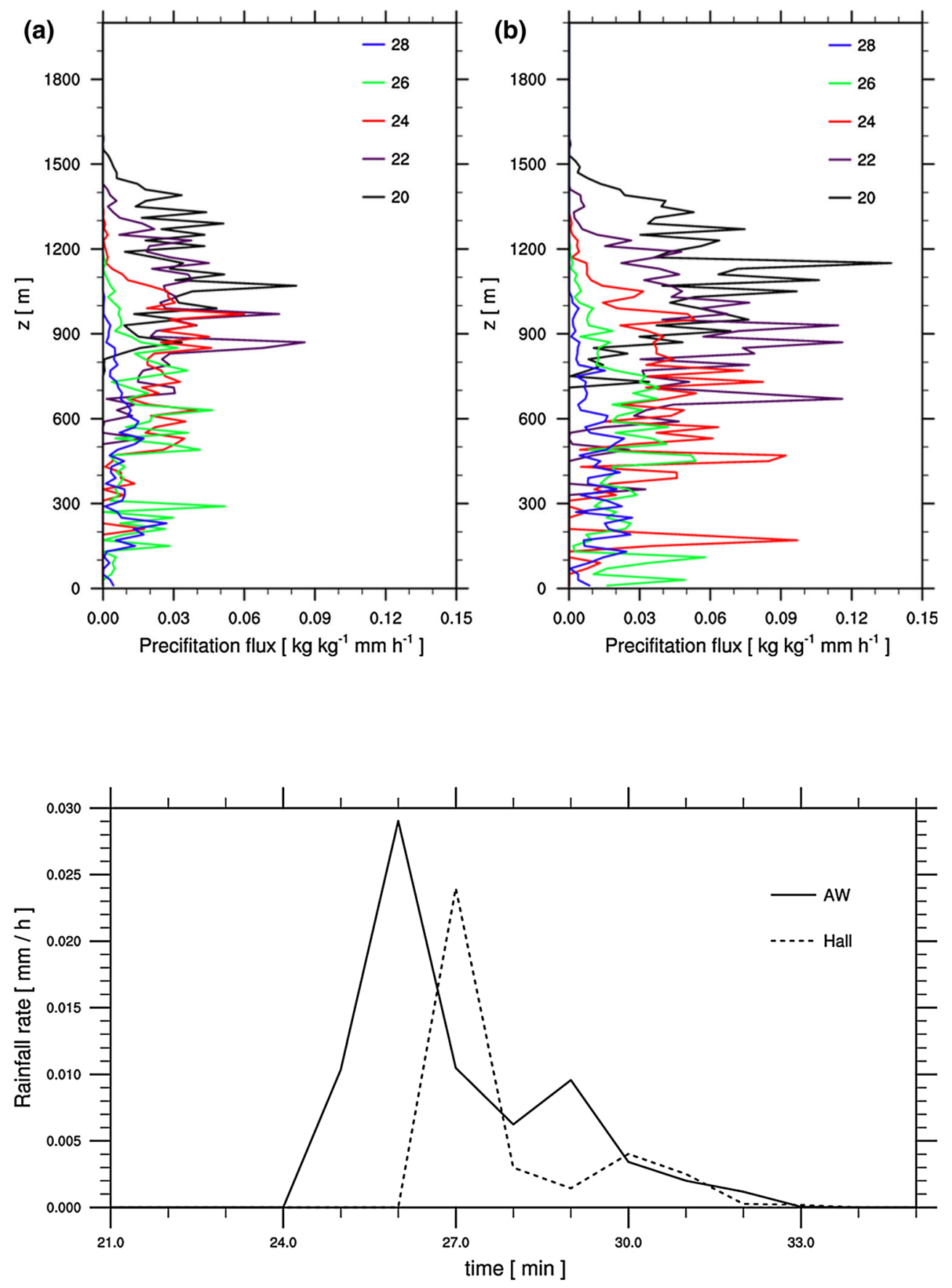
where $n(r)$ is the number of raindrops of size $r$ per unit volume. The precipitation rate $P_{R}$ is then calculated by the average of $\Pi$ over the thickness of $50 \mathrm{~m}$ from the surface.

In bulk models $w_{\mathrm{s}}$ is usually used, instead of $W$, to calculate $\Pi$ in (9). Therefore, it is interesting to investigate how different the value of $\Pi$ obtained in this way is from the real one calculated directly from (9). Figure 13 shows that, if $w_{\mathrm{s}}$ is used for (9), instead of $W$, it becomes substantially smaller than the actual values of $\Pi$. It indicates that the downdraft associated with precipitation, as shown in Figs. 3 and 6, enhances the sedimentation velocity itself. Note that precipitation initiates downdraft by the evaporative cooling of droplets and the downward dragging by settling droplets, although the latter is not considered in the present simulation (e.g., Houze 1993).

Comparison is also made for the profiles of $\Pi$ from two different values of the critical radius $r_{c}\left(r_{c}=40\right.$ and $100 \mu \mathrm{m})$ to investigate its sensitivity. It is found, however, that the critical value of $r_{c}$ does not affect $\Pi$ significantly (Fig. 13). It may be associated with the fact that the droplet mass density is lower and $W$ is smaller for droplets in the range $r=40-100 \mu \mathrm{m}$ than for larger droplets $(r>100 \mu \mathrm{m})$. The use of $w_{\mathrm{s}}$ instead of $W$ can lead to the underestimation of the peak precipitation rate by as much as $28 \%$.

Finally, it is examined how the inclusion of the turbulence effect in the collision kernel affects precipitation by comparing the profiles of $\Pi$ (Fig. 14) and the time series of precipitation rate $P_{R}$ (Fig. 15). It is found that precipitation not only starts earlier but also becomes stronger under the AW kernel, which is consistent with the LES results with the bulk microphysics (Seifert et al. 2010) and with the spectral bin microphysics (Wyszogrodzki et al. 2013). Figure 14 also shows that raindrops fall faster and in a larger amount in the AW scheme than in the Hall scheme, leading to earlier and stronger precipitation, although they appear about the same time in both schemes.

\section{Conclusion}

In the present work, the physical process influencing the microphysical property in a precipitating convective cloud is investigated using the Lagrangian cloud model (LCM), in which the flow field is simulated by LES, and the droplets are treated as Lagrangian particles. For the LCM, the concept of an ensemble of super-droplets, with each super-droplet representing a large number of real droplets of the same size, is introduced, and a statistical method is developed to calculate the growth of a super-droplet by collision/coalescence based on the background droplet spectrum and turbulence, using the collection kernel.
The LCM is shown to reproduce the general features of the cloud and precipitation process successfully, including the evolutions of vertical velocity, liquid-water mixing ratio, dissipation rate, and droplet spectrum. It is then applied to investigate precipitation initiation and to examine the parameterization of cloud microphysics.

It is found that raindrops appear initially near the cloud top and in the downdraft region and tend to cluster with each other. The pattern of simulated precipitation initiation is in good agreement with the observation during RICO by Small and Chuang (2008). The mechanism for the raindrop formation is explained by the fact that, in the entrainment zone near the cloud top, strong turbulence and broadened droplet spectrum are induced by the entrainment of dry air, but high liquid-water mixing ratio is maintained within cloud parts because of insufficient mixing.

Statistical analysis of the vertical velocity of a droplet $W$ reveals that the transition from cloud droplets to raindrops occurs in the range $20 \mu \mathrm{m}<r<100 \mu \mathrm{m}$, while the variation of $W$ depends on turbulence as well as the droplet radius $r$. The general pattern of the raindrop size distribution is consistent with the Marshall-Palmer distribution. The precipitation flux can be underestimated substantially, if the terminal velocity $w_{\mathrm{s}}$ is used instead of $W$, but it is not sensitive to the choice of the critical droplet radius dividing cloud drops and raindrops. It is found that precipitation starts earlier and becomes stronger if the effect of turbulence is included in the collection kernel.

The present work demonstrates that the LCM, which is a more natural approach to simulate particle-laden turbulent flows, can be used to reproduce the realistic dynamic feature of clouds, including precipitation initiation, while providing information of individual droplets that could not be obtained with other models. It thus illustrates that the LCM has a potential to become a powerful tool for understanding the cloud processes and for developing its parameterizations.

Nonetheless, further improvement of the model in various aspects is necessary to simulate more realistic clouds in future. For example, the collision/coalescence scheme and the collection kernel should be further elaborated, possibly by using information from DNS with higher Reynolds numbers, or by comparing with the spectral bin model results. More sophisticated two-phase fluid dynamical processes can be taken into account, such as the effect of the SGS turbulence on the transport of a super-droplet and the exchange of momentum between particle and fluid phases. It is also necessary to investigate further the numerical convergence with increasing resolution and particle numbers under various schemes of SGS turbulence and collision. Finally, there are various other physical processes that must be included for more realistic simulations, such as the activation of $\mathrm{CCN}$ and breakup of large droplets. 
Acknowledgments This work was funded by grants from the National Research Foundation of Korea (MEST; NRF-2009C1AAA001-0093068) and Grant no. ET 8/14-2\&3 and RA 617/25-2 within the SPP 1276 MetStröm program of the German Research Foundation (DFG). LPW acknowledges support from the US National Science Foundation (OCI-0904534, AGS-1139743). All simulations have been carried out on the SGI-ICE systems of the North-German Supercomputing Alliance (HLRN) and the Supercomputing Center/ Korea Institute of Science and Technology Information (KISTI; KSC2011-C3-04).

Open Access This article is distributed under the terms of the Creative Commons Attribution License which permits any use, distribution, and reproduction in any medium, provided the original author(s) and the source are credited.

\section{References}

Andrejczuk M, Reisner J, Henson B, Dubey M, Jeffery C (2008) The potential impacts of pollution on a nondrizzling stratus deck: does aerosol number matter more than type? J Geophys Res 113:D19204

Andrejczuk M, Grabowski WW, Reisner J, Gadian A (2010) Cloudaerosol interactions for boundary layer stratocumulus in the Lagrangian Cloud Model. J Geophys Res 115:D22214

Arabas S, Shima S (2013) Large-eddy simulations of trade wind cumuli particle-based microphysics with Monte Carlo coalescence. J Atmos Sci 70:2768-2777

Armenio V, Piomelli U, Fiorotto V (1999) Effect of the subgrid scales on particle motion. Phys Fluids 11:3030-3042

Ayala O, Rosa B, Wang LP, Grabowski WW (2008a) Effects of turbulence on the geometric collision rate of sedimenting droplets. Part 1. Results from direct numerical simulations. New J Phys 10:075015

Ayala O, Rosa B, Wang LP (2008b) Effects of turbulence on the geometric collision rate of sedimenting droplets. Part 2. Theory and parameterization. New J Phys 10:075016

Baker MB, Corbin RG, Latham J (1980) The influence of entrainment on the evolution of cloud droplet spectra: I. A model of inhomogeneous mixing. Quart J Roy Meteor Soc 106:581-598

Beheng KD, Doms G (1986) A general formulation of collection rates of cloud and raindrops using the kinetic equation and comparison with parameterizations. Beitr Phys Atmos 59:66-84

Brenguier JL, Grabowski WW (1993) Cumulus entrainment and cloud droplet spectra: a numerical model within a two-dimensional dynamical framework. J Atmos Sci 50:120-136

Clift R, Grace J, Weber M (1978) Bubbles, drops, and particles. Academic Press, New York

Cooper WA, Lasher-Trapp SG, Blyth AM (2013) The influence of entrainment and mixing on the initial formation of rain in a warm cumulus cloud. J Atmos Sci 70:1727-1743

Emanuel KA (1994) Atmospheric Convection. Oxford University Press, Oxford

Fede P, Simonin O (2006) Numerical study of the subgrid fluid turbulence effects on the statistics of heavy colliding particles. Phys Fluids 18:04103

Grabowski WW, Wang LW (2009) Diffusional and accretional growth of water drops in a rising adiabatic parcel: effects of the turbulent collision kernel. Atmos Chem Phys 9:2335-2353

Hall WD (1980) A detailed microphysical model within a twodimensional dynamical framework: model description and preliminary results. J Atmos Sci 37:2486-2507

Holland JZ, Rasmusson EM (1973) Measurement of atmospheric mass, energy, and momentum budgets over a 500-kilometer square of tropical ocean. Mon Weather Rev 101:44-55
Houze RA (1993) Cloud Dynamics. Academic Press, New York

Kessler E (1969) On the distribution and continuity of water substance in atmospheric circulations. Meteor Monogr 10(32): $1-84$

Khairoutdinov M, Kogan Y (2000) A new cloud physics parameterization in a large-eddy simulation model of marine stratocumulus. Mon Weather Rev 128:229-243

Kogan YL, Khairudinov MP, Lilly DL, Kogan ZN, Liu Q (1995) Modeling stratocumulus cloud layers in a large eddy simulation model with explicit microphysics. J Atmos Sci 52:2923-2940

Lanotte AS, Seminara A, Toschi F (2009) Cloud droplet growth by condensation in homogeneous isotropic turbulence. J Atmos Sci 66:1685-1697

Lasher-Trapp SG, Cooper WA, Blyth AM (2005) Broadening of droplet size distributions from entrainment and mixing in a cumulus cloud. Quart J Roy Meteor Soc 131:195-220

MacPherson JI, Isaac GA (1977) Turbulent characteristics of some Canadian cumulus clouds. J Appl Meteor 16:81-90

Marshall JS, Palmer WM (1948) The distribution of rain drops with size. J Meteor 5:165-166

Mason BJ (1971) The physic of clouds, 2nd edn. Clarendon Press, Oxford

Mazin I, Silaeva IV, Strunin M (1984) Turbulent fluctuations of horizontal and vertical wind and velocity components in various cloud forms. Izv Atmos Oceanic Phys 20:6-11

Rauber RM et al (2007) Rain in shallow cumulus over the ocean: the RICO campaign. Bull Am Meteor Soc 88:1912-1928

Riechelmann T, Noh Y, Raasch S (2012) A new method for largeeddy simulations of clouds with Lagrangian droplets including the effects of turbulent collision. New J Phys 14:065008

Rogers RR, Yau MK (1989) A short course in cloud physics, 3rd edn. Pergamon Press, Oxford

Seifert A, Beheng KD (2001) A double-moment parameterization for simulating autoconversion, accretion and selfcollection. Atmos Res 59:265-281

Seifert A, Nuijens L, Stevens B (2010) Turbulence effects on warmrain autoconversion in precipitating shallow convection. Quart $\mathbf{J}$ Roy Meteor Soc 136:1753-1762

Shiino JI (1983) Evolution of raindrops in an axissymmetric cumulus model Part I. Comparison of the parameterized and nonparameterized microphysics. J Meteor Soc Japan 61:629-655

Shima S, Kusano SK, Kawano A, Sugiyama T, Kawahara S (2009) The super-droplet method for the numerical simulation of clouds and precipitation: a particle-based and probabilistic microphysics model coupled with a non-hydrostatic model. Quart J Roy Meteor Soc 135:1307-1320

Sidin RSR, Ijzermans RHA, Reeks MW (2009) A Lagrangian approach to droplet condensation in atmospheric clouds. Phys Fluids 21:106603

Siebert $\mathrm{H}$ et al (2006) Probing finescale dynamics and microphysics of clouds with helicopter-borne measurements. Bull Am Meteor Soc $87: 1727-1738$

Siebesma AP et al (2003) A large eddy simulation intercomparison study of shallow cumulus convection. J Atmos Sci 60:12011219

Small JD, Chuang PY (2008) New observations of precipitation initiation in warm cumulus clouds. J Atmos Sci 65:2972-2982

Sölch I, Kärcher B (2010) A large-eddy model for cirrus clouds with explicit aerosol and ice microphysics and Lagrangian ice particle tracking. Quart J Roy Meteor Soc 136:2074-2093

Stensrud DJ (2007) Parameterization schemes: keys to understanding numerical weather prediction models. Cambridge University Press, Cambridge

Uijttewaal WS, Oliemans RV (1996) Particle dispersion and deposition in direct numerical and large eddy simulations of vertical pipe flows. Phys Fluids 8:2590-2604 
Vaillancourt PA, Bartello P, Grabowski WW (2002) Microscopic approach to cloud droplet by condensation. Part II: turbulence, clustering, and condensational growth. J Atmos Sci 59:3421-3435

van Zanten MC et al (2011) Controls on precipitation and cloudiness in simulations of trade-wind cumulus as observed during RICO. J Adv Model Earth Syst 3:M06001

Wang LP, Grabowski WW (2009) The role of air turbulence in warm rain initiation. Atmos Sci Lett 10:1-8

Wang Q, Squires KD (1996) Large eddy simulation of particle-laden turbulent flow. Phys Fluids 8:1207-1223

Wyszogrodzki AA, Grabowski WW, Wang LP, Ayala O (2013) Turbulent collision-coalescence in maritime shallow convection. Atmos Chem Phys 13:8471-8487. doi:10.5194/acp-13-84712013
Xue Y, Wang LP, Grabowski WW (2008) Growth of cloud droplets by turbulent collision-coalescence. J Atmos Sci 65:331-356

Yamamoto Y, Potthoff YM, Tanaka T, Kajishima T, Tsuji Y (2001) Large-eddy simulation of turbulent gas-particle flow in a vertical channel: effect of considering inter-particle collisions. J Fluid Mech 442:303-334

Yeh F, Lei U (1991) On the motion of small particles in a homogeneous isotropic turbulent flow. Phys Fluids A 3:2571-2586

Ziegler CL (1985) Retrieval of thermal and microphysical variables in observed convective storms. Part1: model development and preliminary testing. J Atmos Sci 42:1487-1509 